\title{
Progresos en los aprendizajes, características de los estudios y motivaciones de los estudiantes
}

\author{
Josep M. Masjuan
}

Universitat Autònoma de Barcelona

\section{Resumen}

El presente artículo se plantea hasta qué punto la percepción del éxito en los estudios universitarios por parte de los estudiantes depende de las condiciones en que realizan los estudios y de su propia motivación. Al mismo tiempo, intenta averiguar hasta qué punto las motivaciones de los estudiantes están influidas por la categoría socioprofesional y el sexo. En su parte descriptiva, muestra como se diferencian las distintas áreas de estudio en tres regiones europeas, Baden-Württemberg, Cataluña y Rhône-Alpes, por lo que se refiere a las variables utilizadas para comprobar las dos hipótesis anteriores. Los datos proceden de una encuesta realizada durante el año 2002 en las tres regiones, la cual abarca 4.029 estudiantes universitarios en la mitad de sus estudios.

Palabras clave: progreso académico de los estudiantes, universidad, origen social, género.

Abstract. Progresses in the learnings, characteristics of the studies and motivations of the students

This article analyses to what extent the perception of success in university studies by the students depends on the conditions in which they carry out their studies and on their own motivation. At the same time, it attempts to find out to what extent the students' motivations are influenced by socio-professional category and sex. In its descriptive part, this paper shows how the various areas of the study are differentiated in three European regions: Baden-Württemberg, Catalonia and Rhône-Alpes; this is why it refers to the variables used to verify the two previous hypotheses. The data come from a survey carried out in the year 2000 in these three regions. This survey includes 4,029 university students halfway through their studies.

Key words: students performance, university, social origen, gender.

\section{Sumario}

1. Introducción

2. Metodología

3. Descripción de las variables fundamentales
5. Discusión de los resultados y conclusiones

Apéndice

Bibliografía

4. Contraste de hipótesis 


\section{Introducción}

Las sociedades europeas sufren un proceso de globalización económica que, habiéndose iniciado a partir de las crisis de las formas de regulación social de mediados de los años setenta, ha evolucionado en espiral fundamentalmente a partir de la difusión de las nuevas tecnologías de la información. Este proceso afecta a todos los terrenos de la vida social, lo que significa un proceso de cambio rápido y relativamente imprevisible que tiene consecuencias sobre todas las instituciones sociales y, por consiguiente, afecta a los procesos de socialización y de construcción de la propia identidad de las nuevas generaciones. Son difíciles de prever los procesos concretos, debido a su complejidad y a la reflexividad propia de las acciones humanas.

Las nuevas tecnologías de la información producen en la actualidad un cambio en las formas de organización del trabajo y en consecuencia en las formas de ejercicio profesional de los graduados universitarios. Al mismo tiempo, la aplicación de estas nuevas tecnologías en los contextos de aprendizaje cambiará en breve las formas tradicionales de organizar la misma universidad (Barnett, 2000).

Las tasas de participación de los jóvenes de ambos sexos en la enseñanza superior no ha cesado de aumentar en los últimos años en los países desarrollados de Europa, lo que ha producido un aumento en el número de efectivos. Los cambios demográficos que experimentan algunos países como España, reducirán quizás el número total de efectivos, pero en ningún caso es previsible una caída significativa de la tasa de participación de la población joven en las instituciones universitarias. Este aumento constante de las tasas de participación ha comportado lógicamente un aumento de la diversidad de los estudiantes en lo que hace referencia al sexo, al origen social, a los contextos de socialización primaria y por consiguiente a materia de motivaciones y expectativas (Béduwé, Planas, 2002). Cada día hay más estudiantes que desean cursar sus estudios a tiempo parcial combinándolos con el trabajo remunerado o bien que desean asistir a la universidad una vez han conseguido una primera inserción laboral, y que prefieren una participación semipresencial o totalmente virtual acompañados por una orientación adecuada. En la organización de los planes de estudio, diferentes combinaciones entre la disciplinariedad y la profesionalización se van imponiendo en diversos países europeos.

Las dificultades de inserción en el mercado laboral de la juventud, incluida la universitaria, ha influido en la imagen y en las expectativas que ésta tiene de la utilidad de la universidad de cara a conseguir una buena inserción profesional con cierta rapidez y, por tanto, en las actitudes y prácticas que desarrolla mientras cursa los estudios, entre las cuales la compaginación de los estudios con el trabajo remunerado, con el objetivo de estar presente en el mercado laboral antes de acabar los estudios para facilitar así su inserción posterior (Mc Innis, 2002; Masjuan y otros, 2002; Aqu Catalunya, 2003).

Una de las consecuencias más evidentes de las tendencias anteriormente indicadas consiste en que la universidad debe aumentar considerablemente su 
preocupación por los estudiantes. Esta preocupación reclama frecuentemente un cierto cambio de inercias, ya que la cultura académica está muy fundamentada en las disciplinas científicas, en la importancia de la investigación e incluso en una cierta concepción elitista sobre los estudiantes a los que se les atribuye espontáneamente los mismos objetivos y motivaciones que las del profesorado.

Incluso más recientemente, cuando los gestores universitarios se preocupan de los estudiantes lo hacen más en función de los intereses de la institución que guiados por una preocupación directa en los jóvenes que forman parte de la universidad (Johnson y Deem, 2003).

En este contexto, el conocimiento regular de la población estudiantil, de sus características, motivaciones y expectativas, parece un objetivo prioritario para facilitar una consciencia adecuada de sí misma a la propia comunidad universitaria, con la finalidad de ajustar constantemente su funcionamiento al servicio de las personas directamente implicadas en la institución y cumplir así lo más eficazmente posible los objetivos que la sociedad democrática atribuye a la universidad.

Ha sido en este marco que tres regiones europeas, Cataluña, BadenWürttemberg y Rhône-Alpes, organizadas en la FREREF (Federación de las Regiones de Europa para la Investigación en Educación y Formación) han llevado a término una investigación sobre los estudiantes universitarios de las mismas. El objetivo de este artículo es presentar los resultados de alguno de los aspectos abordados en dicha investigación.

\section{El éxito en los aprendizajes como una función de las condiciones de estudio y de la motivación}

Asumimos en este artículo que el aprendizaje de los estudiantes puede ser considerado como un cambio de estado en su personalidad y que puede ser explicado por dos factores principales que se derivan de aplicar el modelo de Herzberg (1959), que fue pensado para analizar el cambio planificado en el mundo del trabajo, a la situación de estudiar en la universidad. El autor denominó a estos dos factores «higiene y motivación» y considera que ambos son percibidos por los individuos como independientes el uno del otro. La higiene se refiere a las condiciones de trabajo, es decir, a cómo los individuos perciben el ambiente de trabajo y la calidad del mismo, y la motivación, a la implicación intelectual y emocional con el mismo, es decir, a las emociones internas que emergen en los individuos a partir de la realización de un determinado trabajo y que son intrínsecas al mismo. Aplicado el modelo al proceso de aprendizaje, las condiciones de trabajo se refieren a la buena organización y al funcionamiento del sistema universitario, y la motivación, a la implicación emocional e intelectual de los estudiantes por el hecho de considerarlo una experiencia interesante para su trayectoria vital (Cryer y Elton, 1990; Elton, 1996).

Así pues, asumimos como hipótesis fundamental de nuestro trabajo que el éxito en los estudios es una función de la percepción de las propias condiciones 
en que se realiza la tarea de estudiar y de las motivaciones internas del propio estudiante.

\section{La motivación en los estudios como una función del origen social y el sexo}

Para aproximarnos a la problemática de la motivación, hemos partido de las dos dimensiones clásicas: las motivaciones expresivas y las motivaciones instrumentales (Fernández Enguita, 1988). La primera recoge como motivos del ingreso en la universidad el interés por las disciplinas de estudio y la confianza en las propias capacidades, y el segundo, las oportunidades de promoción profesional.

Existe un acuerdo generalizado entre los sociólogos que los procesos de socialización en la infancia generan hábitos diferentes según la clase social de pertenencia que están relacionados con las formas de organización familiar, los estilos educativos y la capacidad de acceso a los recursos económicos y culturales (Bernstein, 1990; Bourdieu, 1969). La socialización de los niños y las niñas es también diferente en todos los contextos culturales y adquiere matices importantes según las tradiciones familiares, muchas veces asociadas a la clase social. Este tipo de hábitos, muchas veces inconscientes, estructuran las preferencias y motivaciones de los sujetos sociales y, por consiguiente, contribuyen a la definición de la propia identidad.

El predominio de las estrategias instrumentales debería ser más frecuentes en los alumnos procedentes de las clases populares, puesto que los valores de su socialización primaria son distintos de los de la cultura académica y en muchos casos estos individuos pueden haber sufrido una fuerte presión familiar para acudir a la universidad, considerada como un camino de promoción o como un mecanismo de defensa frente a dificultades en la inserción profesional. Contrariamente, entre los estudiantes procedentes de las clases medias profesionales y técnicas deberían predominar las motivaciones expresivas o una combinación de ambas, como resultado más coherente de su socialización en un entorno más semejante al universitario.

Entre las mujeres debería esperarse una fuerte presencia de las motivaciones instrumentales, puesto que los valores expresivos que muchas de ellas han recibido todavía en la socialización primaria no se corresponden con la valoración del estudio y la profesión. Las jóvenes acudirían a la universidad respondiendo a la presión social y a su propia percepción de la necesidad de cambiar su propio papel en la sociedad.

Las afirmaciones anteriores, que se refieren al aumento de la diversidad de los estudiantes a partir del crecimiento de los efectivos, pueden ser compensadas por las presiones hacia la homogeneidad que se generan al mismo tiempo en las sociedades contemporáneas. Los rápidos cambios sociales que se producen en la actualidad han modificado significativamente la estructura de clases y al mismo tiempo la influencia de los medios de comunicación han modificado los valores culturales asociados a las mismas, lo cual actúa a favor de una cierta homogeneización cultural. Aportar elementos para esclarecer la 
importancia de ambas tendencias forma parte del objetivo de nuestra investigación.

Así pues, las hipótesis principales de la investigación presentada en este artículo son las siguientes:

a) Hasta qué punto los progresos en el aprendizaje dependen de la percepción subjetiva de las condiciones en el estudio y de la motivación.

b) Hasta qué punto la motivación hacia los estudios depende del sexo y de la clase social.

El artículo se organiza en cuatro partes fundamentales: en la primera de ellas se explícita la metodología utilizada; en la segunda se describen las principales variables utilizadas distinguiendo las tres regiones europeas y las áreas en las que se han agrupado las distintas titulaciones, haciendo muchas referencias al apéndice en el cual se transcriben los datos básicos para comprender adecuadamente las dimensiones tratadas en el estudio; en la tercera parte se contrastan las hipótesis antes indicadas, y en la última se muestran las conclusiones y se discuten los resultados obtenidos ${ }^{1}$.

\section{Metodología}

Los datos que siguen corresponden a una investigación sobre los estudiantes universitarios realizada en tres regiones europeas: Baden-Württemberg, Cataluña y Rhône-Alpes, en el marco de la red UNI21 de la FREREF (Fédération des Régions d'Europe sur la Recerche en Éducation et Formation) (Hadji y otros 2003; Masjuan y otros, 2003).

Se trata de una encuesta realizada a 4.029 estudiantes cuando se están en la mitad de sus estudios, teniendo en cuenta las distintas áreas ${ }^{2}$, pero sin seguir criterios estrictos de muestreo para dar más peso a las titulaciones más consolidadas dentro de las distintas áreas. Del total de cuestionarios, 984 corresponden a Baden-Württemberg; 1.230, a Rhône-Alpes, y 1.823, a Cataluña.

Para medir la percepción de las condiciones en el estudio, hemos utilizado una pregunta que pretende recoger la percepción que los mismos estudiantes tienen del carácter de la carrera que están cursando. Nos ha parecido imprescindible considerar como variables de control dos aspectos objetivos que sin duda tienen que ver con los resultados académicos y que forman parte de las condiciones del estudio en sentido amplio: por una parte, la participación de los alumnos en el mercado laboral, puesto que en la mayoría de los casos resta tiempo para poderlo dedicar al estudio, y las aptitudes de los estudiantes medidas con las notas de acceso a la universidad, puesto que

1. Pueden consultarse los informes regionales de Cataluña y Rhône-Alpes (Hadji y otros, 2003; Masjuan y otros, 2003).

2. Las áreas corresponden a agrupaciones de titulaciones semejantes. 
también afectan a la mayor o menor dificultad asociada con la actividad de estudiar ${ }^{3}$.

Para medir la motivación hacia los estudios y la percepción del éxito en los mismos, se han utilizado dos preguntas con variables construidas para este propósito. La descripción concreta de cada una de estas variables se encuentra en el apéndice.

Hemos utilizado el análisis de componentes principales para reducir el número de variables y hacerlas más consistentes y el análisis de la varianza y la regresión múltiple para establecer el contraste de nuestras hipótesis. Indicamos con un asterisco $\left(^{*}\right)$ las relaciones que tienen una significación estadística por los menos al 95\% de probabilidad. Para medir la fuerza de la relación con variables de atributo, hemos utilizado el coeficiente de contingencia.

El análisis que se presenta en este artículo se basa exclusivamente en los datos cuantitativos de la encuesta reseñada. En el proyecto original, se proponía complementariamente una aproximación cualitativa que se ha empezado a realizar y el presente número de PAPERS contiene una primera muestra de ella.

\section{Descripción de las variables fundamentales}

En este apartado, nos referimos a las distintas variables consideradas, diferenciándolas según las regiones y las áreas de estudio con una finalidad descriptiva.

\section{Las motivaciones de los estudiantes}

El cuestionario utilizado contiene siete indicadores que, a partir de un análisis de componentes principales, fueron reducidos a tres ejes, que responden perfectamente a los presupuestos teóricos tenidos en cuenta en la construcción del cuestionario. Transcribimos en el apéndice los tres factores con los indicadores y los parámetros asociados a cada uno.

Los tres factores son los siguientes:

- Motivaciones expresivas: interés específico por la disciplina de estudio y aptitudes.

- Motivaciones profesionales: opción estrechamente relacionada con una profesión.

- Motivaciones instrumentales: expectativas de buenos ingresos, perspectivas de obtener un trabajo seguro, diversidad de oportunidades profesionales y buenas perspectivas de llegar a ser un ejecutivo.

3. Las calificaciones de acceso no responden al mismo procedimiento en las tres regiones, sin embargo, los equipos investigadores han realizado un esfuerzo de homogeneización con los datos disponibles. 

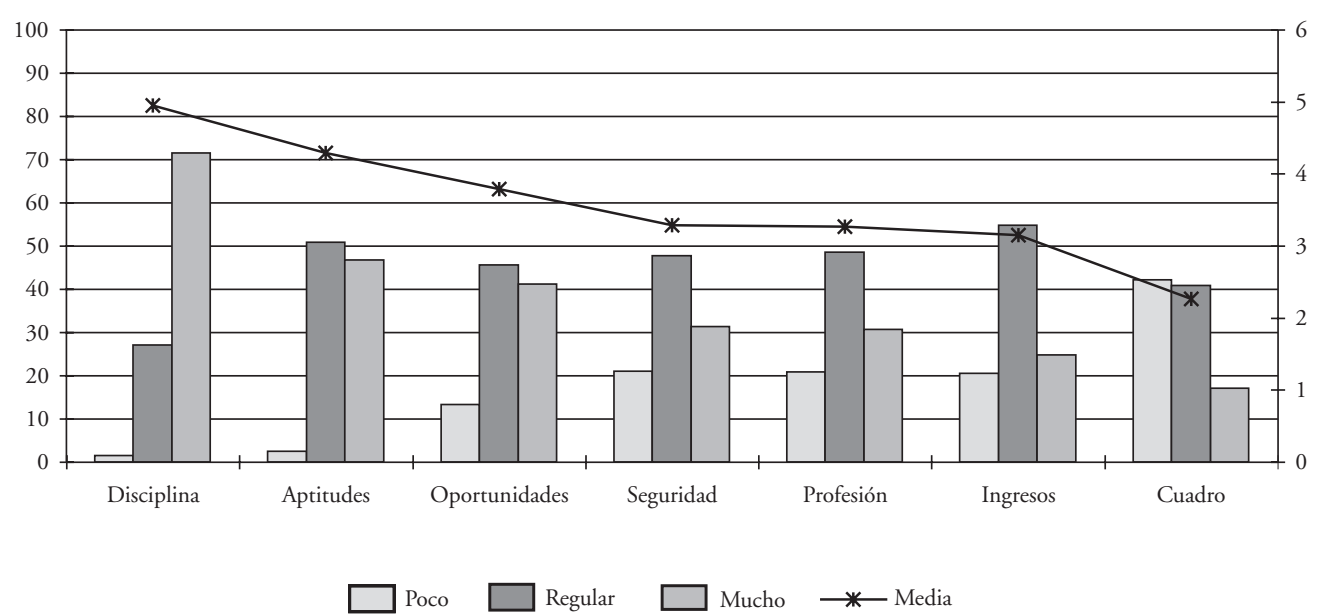

Gráfico 1. Motivaciones de los estudiantes.

El análisis factorial muestra que el interés específico por la materia de estudio correlaciona negativamente con el factor que recoge el conjunto de indicadores que expresan un interés instrumental, lo cual indica con claridad la diferencia entre las preferencias que existen entre los estudiantes que acuden a la universidad. En cambio, el indicador que se refiere directamente a la profesión no correlaciona con el factor expresivo y correlaciona positivamente con el factor instrumental, es decir que la elección de la profesionalidad es como una situación intermedia en la que se pueden combinar, según los casos, los motivos instrumentales y los expresivos. En los análisis posteriores a este apartado, hemos preferido utilizar los dos factores claramente contrapuestos que corresponden a los motivos expresivos y a los instrumentales.

El gráfico 1 nos muestra ordenadamente el conjunto de los indicadores referidos a las motivaciones de los estudiantes.

Podemos observar que las medias más altas corresponden a los motivos expresivos, lo cual indica que la gran mayoría de estudiantes en las tres regiones hacen la opción de los estudios fundamentalmente porque les gusta la materia y se perciben con capacidades para estudiarla.

En último término, encontramos la seguridad en el empleo y la opción que se refiere a las posibilidades de llegar a ser un cuadro en la empresa. Es interesante remarcar que el valor de seguridad, en otros tiempos muy importante, ha dejado de ocupar las primeras preferencias de los estudiantes, quizás a partir de un mecanismo de adaptación a la situación.

Los gráficos 2, 3 y 4 muestran las diferencias entre áreas de estudio por cada una de las regiones estudiadas. 


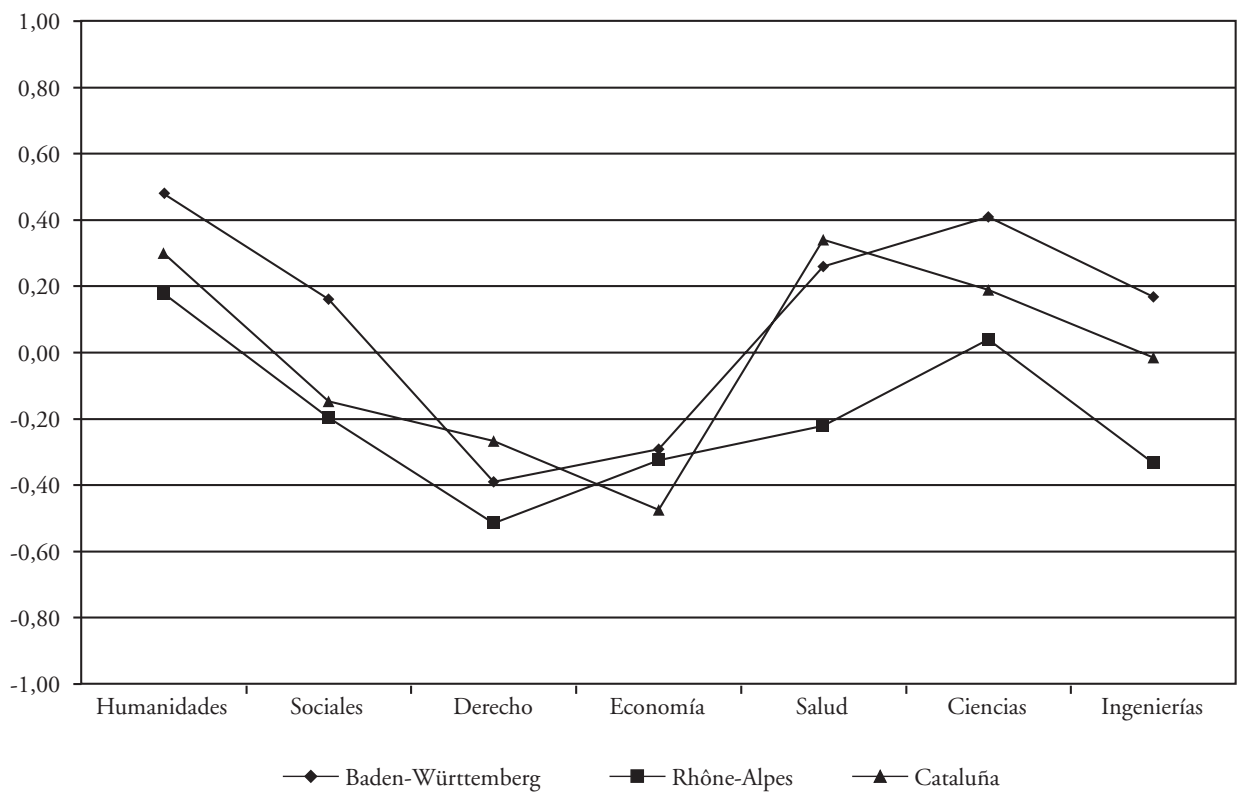

Gráfico 2. Motivos expresivos.

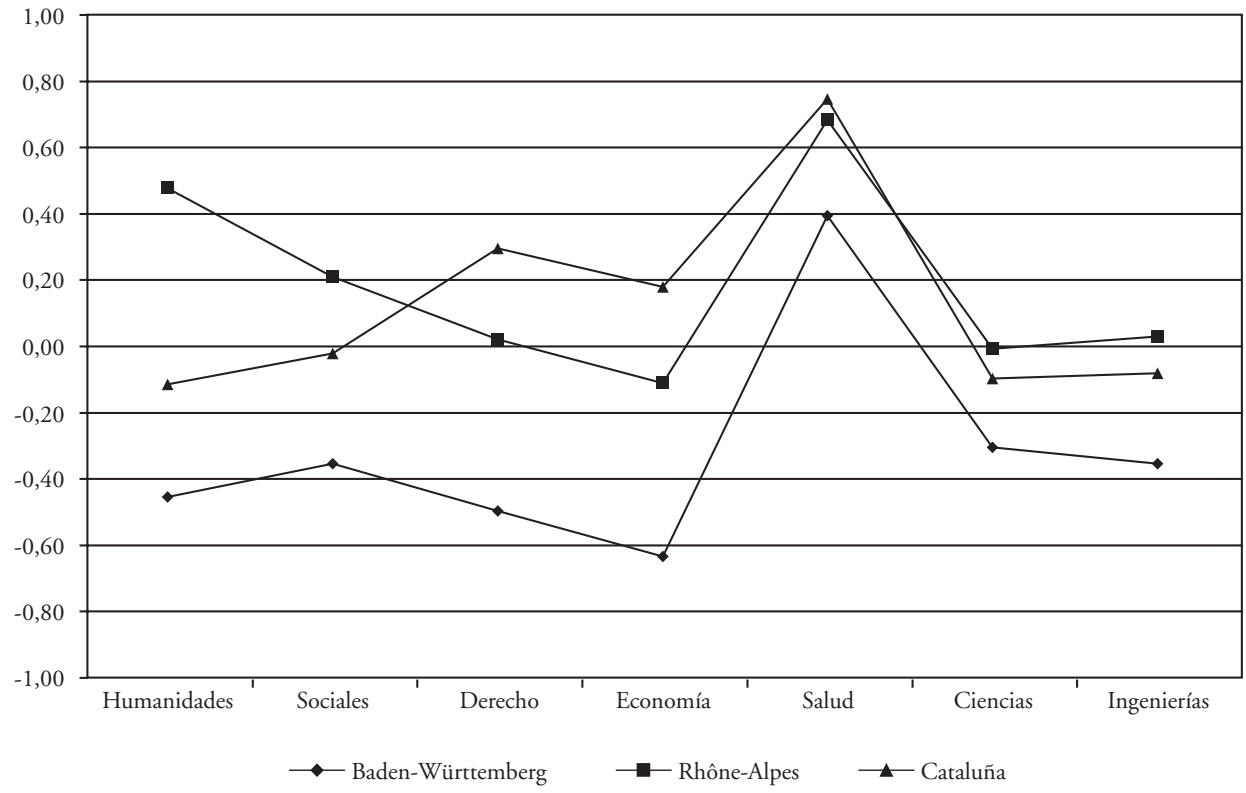

Gráfico 3. Motivos profesionales. 


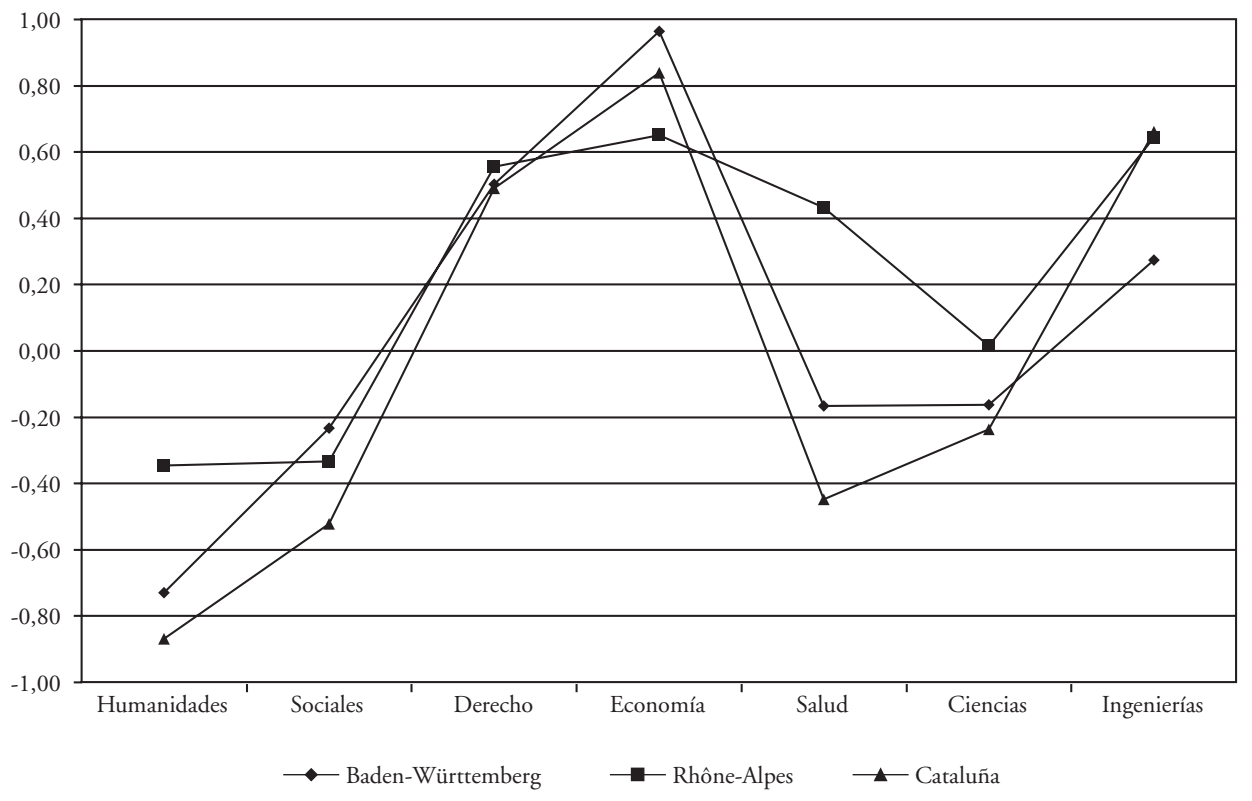

Gráfico 4. Motivos instrumentales.

En los tres casos, las diferencias son significativas por área de estudio, pero son más grandes en la motivación instrumental que en la motivación profesional y la motivación expresiva.

Los gráficos nos muestran que, aunque existen diferencias entre regiones, los perfiles de las distintas áreas mantienen una fuerte similitud, lo cual nos indica que, más allá de las culturas propias de cada región, las connotaciones de cada una de las áreas se mantienen constantes.

Los resultados muestran que las áreas de Economía, Derecho e Ingeniería se contraponen a las de Humanidades, Sociales, Salud y Ciencias, ya que, mientras las primeras prefieren los valores instrumentales, las segundas se decantan por los expresivos. El área de Salud es la que más destaca por su preferencia por los aspectos profesionales de los estudios, pero también mantiene una elevada puntuación en los valores expresivos. Así, por ejemplo, mientras un 86\% de los estudiantes del área de Salud y un 83\% de los del área de Humanidades eligen los estudios por interés directo en la materia, solamente un $55 \%$ de los estudiantes de Derecho y un 51\% de los de Economía hacen la misma opción ${ }^{4}$. En el caso de las motivaciones instrumentales, tenemos que mientras un $74 \%$ de los estudiantes de Derecho y Economía eligen sus estudios por las oportu-

4. Los porcentajes se refieren a las dos primeras respuestas en una escala de siete. 
nidades profesionales que ofrecen, solamente un $18 \%$ de los de Humanidades, un $26 \%$ de los de Sociales y un 31\% de los de Salud lo hacen con esta motivación. Las otras áreas tienen proporciones intermedias, tal como se refleja en el gráfico.

Desde el punto de vista de las regiones, destaca la región alemana por su tendencia a puntuar menos en todas las áreas en los motivos estrictamente profesionales, probablemente porque en este país la enseñanza más profesionalizada se realiza fuera de la universidad. En este sentido, excluyen totalmente la motivación profesional un $30 \%$ de los estudiantes de Baden-Württemberg frente a un $18 \%$ de los de Rhône-Alpes y Cataluña 5 .

\section{Las condiciones de trabajo en la universidad}

El cuestionario contiene once indicadores con diferentes aspectos que pueden caracterizar a los diferentes estudios universitarios, los cuales fueron valorados por los encuestados en una escala de 0 a 6 . Los indicadores utilizados son los siguientes:

- Una orientación especializada.

- Mucha exigencia.

- Estudios bien estructurados.

- Claridad en las exigencias en relación con los exámenes.

- Relaciones entre la enseñanza y la investigación.

- Una buena preparación con la profesión y relación estrecha con la práctica.

- La introducción de nuevos métodos didácticos.

- Competitividad entre los estudiantes.

- Buenas relaciones de los estudiantes con los profesores y las profesoras.

- Los estudiantes de la titulación están en desventaja en relación con otros estudiantes.

- Cursos y seminarios con demasiados alumnos y alumnas.

El gráfico 5 nos muestra los once indicadores ordenados a partir de la media aritmética en el conjunto de la muestra estudiada.

$\mathrm{El}$ aspecto que mejor caracteriza a los estudios según los estudiantes es su exigencia, ya que se trata del único aspecto que llega a una puntuación media de 4; entre los aspectos que superan al 3, valor central teórico de la distribución, cabe destacar la buena relación entre el profesorado y los estudiantes, la especialización de los estudios, la buena estructuración y la claridad en las exigencias en los exámenes. Pocos estudiantes en conjunto consideran que su campo de estudio esté en desventaja, cuestión que está cerca de uno en BadenWürttemberg y Rhône-Alpes y en cambio de 2,5 en Cataluña. Así, mientras un $78 \%$ de los estudiantes de la región alemana y un $72 \%$ de la francesa no consideran que sus estudios estén en desventaja en relación con otros, solamente

5. La exclusión se refiere a las dos últimas opciones en una escala de siete. 


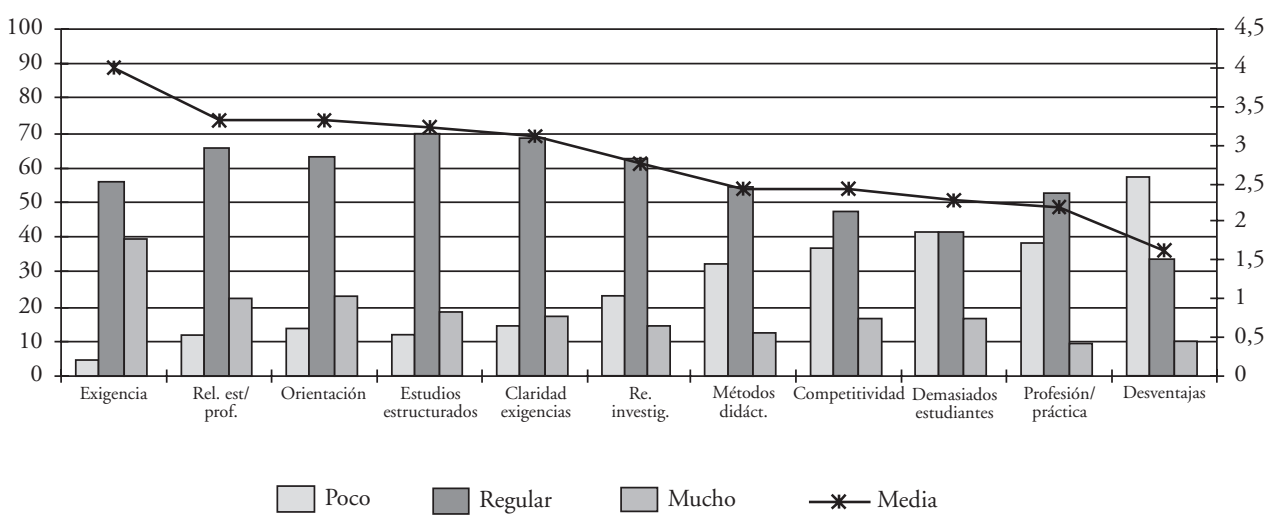

Gráfico 5. Caracterización de los estudios universitarios.

un 35\% de los estudiantes catalanes opina lo mismo. Los resultados muestran que esta diferencia se debe, principalmente, a cuestiones de masificación.

Para facilitar el análisis, hemos realizado un análisis de componentes principales que ha reducido los 11 indicadores a 3 factores que en total resumen el $50 \%$ de la varianza de los 11 indicadores.

El primer factor, que denominamos «orientación profesionalizadora», agrupa los siguientes indicadores: buena preparación para una profesión, introducción de nuevos instrumentos didácticos, buenas relaciones con los profesores, relaciones entre la investigación y la docencia y claridad en las exigencias de los exámenes.

El segundo factor que, denominamos «orientación especializada», agrupa los siguientes indicadores: mucha exigencia, estudios bien estructurados y estudios especializados.

El tercer factor, que denominamos «estudios con desventajas», agrupa los siguientes indicadores: estudiantes en situación de desventaja, estudios masificados, competitividad entre estudiantes.

Los aspectos más destacables del análisis factorial son, por una parte, que los estudios más especializados y exigentes y los que los estudiantes consideran que están en desventaja correlacionan negativamente con la buena relación entre profesores y docentes. Por otra parte, los estudios en desventaja correlacionan negativamente con la buena estructuración de los estudios y la claridad en los exámenes. En definitiva, los alumnos perciben una mejor relación con el profesorado en aquellos estudios más profesionalizados, con medios didácticos distintos y con menos énfasis en la exigencia; al mismo tiempo, las situaciones de desventaja no se refieren solamente a la masificación, sino también a los aspectos organizativos de las titulaciones.

A continuación analizamos las diferencias en cada uno de los tres factores según el área de estudio y la región (gráficos 6, 7 y 8). 


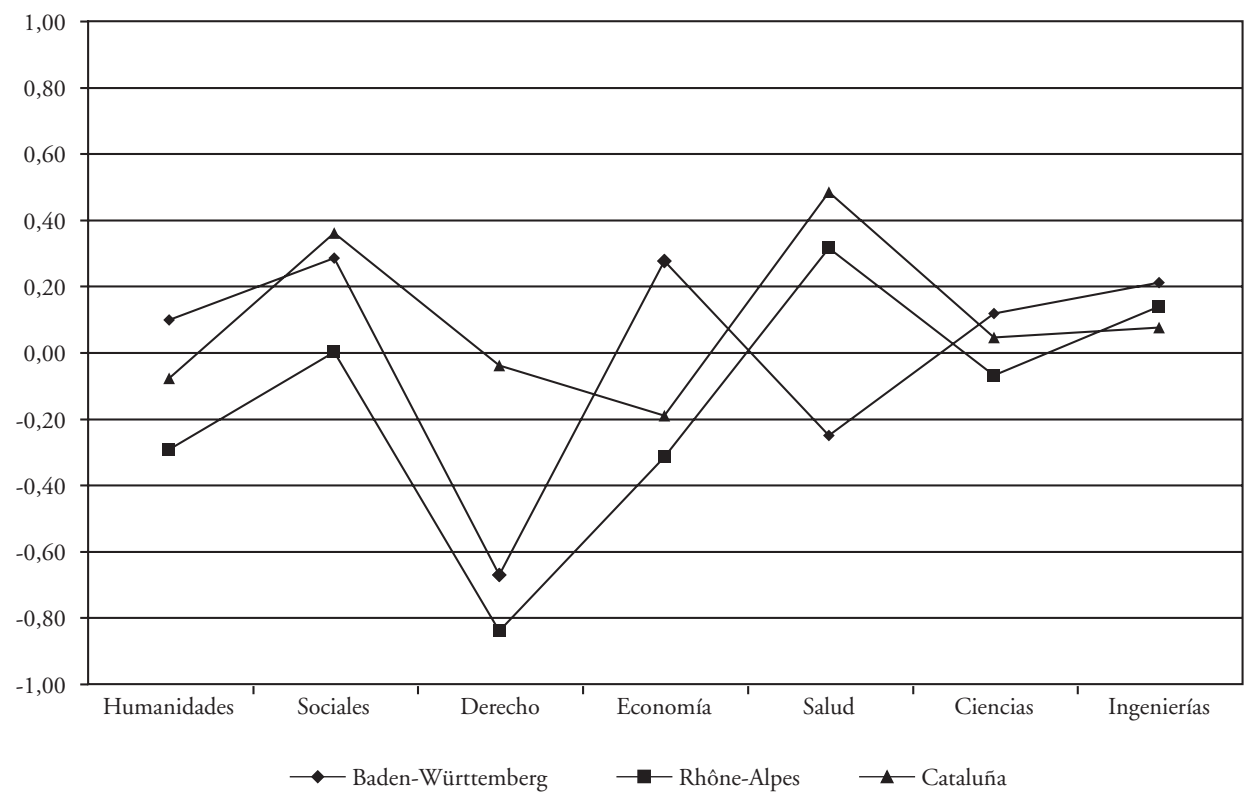

Gráfico 6. Orientación profesionalizadora.

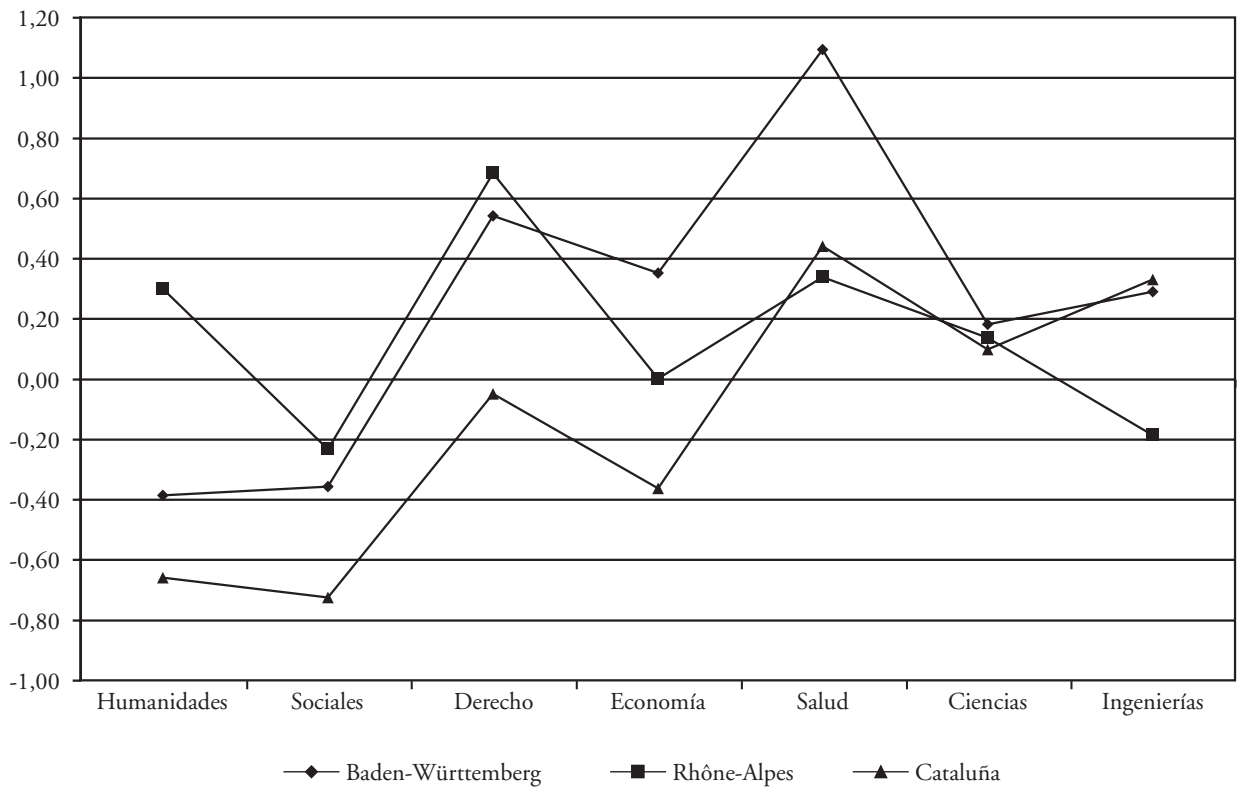

Gráfico 7. Orientación especializada. 


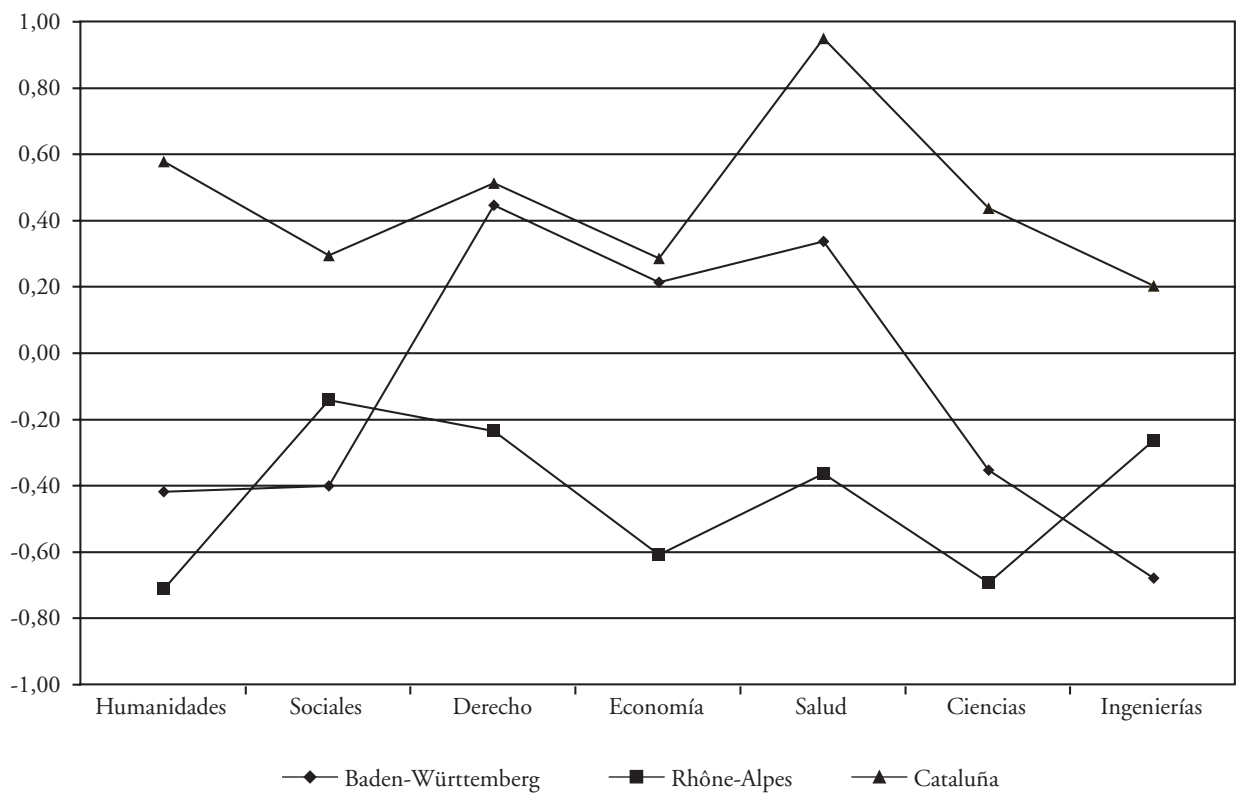

Gráfico 8. Estudios con desventajas.

Destaca el área de Derecho, sobretodo en Rhône-Alpes y BadenWürttemberg, en el factor que hemos denominado «orientación profesionalizadora», por su puntuación media negativa en este factor, lo cual indica que se trata en estas regiones de unos estudios más bien teóricos, con poca innovación didáctica y con poca relación entre los estudiantes y los profesores. Es relevante este hallazgo si tenemos en cuenta que los estudios de Derecho están orientados precisamente hacia el ejercicio de la profesión de abogado.

Así pues, a manera de ejemplo, mientras la poca orientación práctica y profesional de los estudios de Derecho es subrayada por un $51 \%$ de los estudiantes de Baden-Württemberg frente a un $41 \%$ de esta región que tienen esta opinión en el conjunto de las áreas, en la región francesa los porcentajes son, respectivamente, $49 \%$ y $41 \%$ y en Cataluña, $28 \%$ y $34 \%$. Es decir, que únicamente en Cataluña la orientación más práctica del área de Derecho se asemeja al conjunto de las áreas de la región situándose por encima de las otras dos regiones.

Las áreas de Ciencias e Ingeniería presentan una gran similitud interregional situándose próximas a la media, y las áreas de Sociales, que también se asemejan, ocupan una posición positiva en Baden-Württemberg y Cataluña.

Las áreas de Economía y Salud presentan mayor dispersión y, por consiguiente, es más difícil de hablar de una característica común entre las tres regio- 
nes. Cataluña y Rhône-Alpes en el área de Salud y Baden-Württemberg en el área de Economía quedan mejor situadas.

En el factor denominado "orientación especializada» podemos observar, mejor que en el caso anterior, la influencia de las áreas de estudio; aún existiendo diferencias significativas entre regiones, la lógica de las diferentes áreas se manifiesta claramente. Así pues, vemos como las áreas de Derecho y de Salud se muestran como las más especializadas y exigentes y en el polo opuesto se sitúa el área de Sociales. En los tres países, las áreas de Ciencias aparecen próximas a la media y las áreas de Economía, Humanidades e Ingeniería presentan mayor dispersión.

Comparando este gráfico con el anterior, se puede observar que el área de Salud es la que destaca por compaginar la orientación profesional y práctica con la orientación especializada y exigente. De todas maneras, también se da la contraposición en el caso de la región alemana.

Cataluña es la región que muestra más tendencia a tener «estudios con desventajas» frente a Rhône-Alpes, que es la región mejor situada.

Destaca el área de Salud en Cataluña en este factor; así pues, los estudiantes consideran que no están en las mejores condiciones para cursar unos estudios que requieren trato más personalizado. En la región alemana, Derecho, Economía y también Salud se diferencian de las otras áreas de la región en este aspecto.

Así pues, a manera de ejemplo, mientras el porcentaje de alumnos que consideran que sus estudios están en desventaja son un $18 \%$ en Cataluña, éste es de un $4 \%$ en la región francesa, y de un $2 \%$ en la alemana. Si nos fijamos en el indicador que se refiere a la masificación, los porcentajes de estudiantes que consideran que sus estudios están muy masificados son del $21 \%$ en BadenWürttemberg, del 18\% en Cataluña y del 12\% en Rhône-Alpes.

\section{Las calificaciones al ingresar en la universidad}

Tal como hemos indicado en el apartado sobre metodología, las notas al ingresar en la universidad son un indicador de las aptitudes de los estudiantes, condicionan la mayor o menor dificultad para el estudio y, por consiguiente, se refieren de manera indirecta a las condiciones de trabajo en la universidad.

Los procedimientos para establecer estas calificaciones son distintos según los países, pero se ha hecho un esfuerzo de homogeneización para agrupar los datos en las tres categorías que se muestran en el gráfico 9.

Según estos resultados, la universidad alemana sería, en general, más exigente a la hora de seleccionar a los estudiantes.

En los tres países, la titulación de Medicina es la que demanda unos estudiantes con unas aptitudes más altas. 


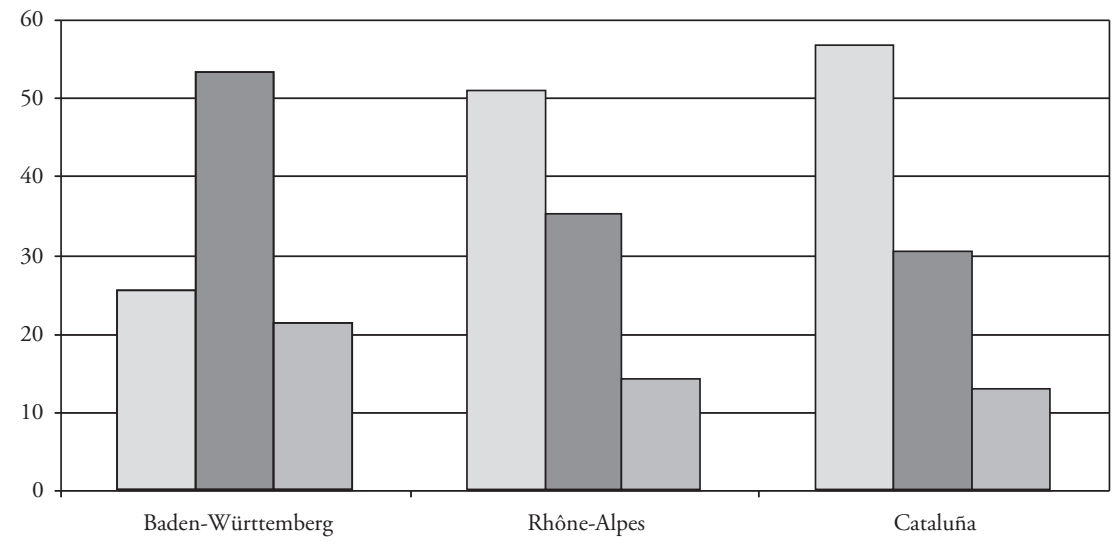

Gráfico 9. Calificaciones de ingreso en la universidad por regiones.

\section{La compaginación de los estudios con el trabajo remunerado}

Uno de los aspectos que influyen en la concepción que los estudiantes tienen de la universidad es la compaginación de los estudios con el trabajo remunerado, que, según diversos autores, tiende a aumentar. Se trata de un aspecto que sin duda afecta a las condiciones de estudio, en tanto que reduce la presencia en la universidad y limita las posibilidades de dedicación al estudio.

A través de un indicador con cuatro categorías, se ha intentado captar esta realidad objetiva de los estudiantes en dos de las tres regiones consideradas ${ }^{6}$.

Como puede verse en el gráfico 10, la compaginación de los estudios con el trabajo remunerado es mucho más frecuente en Cataluña que en la región de Rhône-Alpes, tanto si consideramos los que tienen propiamente hablando una ocupación, con más o menos dedicación, como si tenemos en cuenta los que realizan pequeños trabajos de acompañamiento de estudios.

La dedicación a los estudios a tiempo completo es más frecuente en las áreas de Salud, Ciencias e Ingeniería, en cambio, los economistas y los ingenieros son los que más tiempo dedican al trabajo remunerado ${ }^{7}$.

6. La región de Baden-Württemberg no incluyó esta pregunta en el cuestionario. A partir de otro indicador, se puede estimar que los estudiantes a tiempo completo se sitúan entorno al $62 \%$.

7. Existe una relación muy débil entre el origen social y la compaginación entre los estudios y el trabajo. El origen social, además, no es una variable que influya mucho en las actitudes y en los comportamientos de los estudiantes, lo cual puede interpretarse como una generalización de la condición estudiantil y su estilo de vida (Masjuan, Troiano y Molins, 2003). 


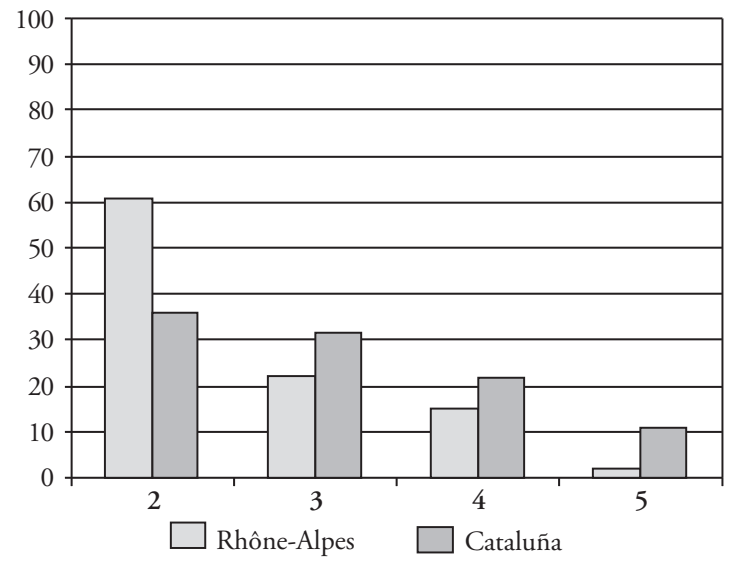

Gráfico 10. Compaginación de los estudios con el trabajo por regiones.

2: estudiantes a tiempo completo; 3: pequeños trabajos complementarios; 4: ocupación a tiempo parcial; 5: ocupación de más de media jornada.

Los estudiantes que realizan pequeños trabajos de complemento de estudios dedican al trabajo, como promedio, 6 horas a la semana, los que tienen una ocupación a tiempo parcial, 13,5 horas semanales y los que trabajan más de media jornada, 31 horas semanales, siendo en los dos últimos casos superior la dedicación en Cataluña (gráfico 11$)^{8}$.

Estos resultados indican claramente las dificultades de los estudiantes que tienen una ocupación, más allá de un simple trabajo de acompañamiento, para asistir regularmente a clase e incluso en muchos casos para poder dedicar algunas horas al estudio.

La razones que aducen los estudiantes que realizan trabajos remunerados para justificar su dedicación al mismo son las siguientes (suma de los dos valores más altos en una escala de siete):

- Financiación del ocio: 44\%.

- Interés por hacer algo práctico: $36 \%$.

- Prepararse para la profesión futura: 35\%.

- Financiar los estudios: 31\%.

- Aumentar las oportunidades de inserción: 29\%.

Estos datos son algo diferentes según la regiones. Destaca el caso de Cataluña, que tiene el porcentaje más bajo de «financiación de los estudios» $(21 \%)$ y en

8. El gráfico se ha calculado controlando la variable área de estudio. Los resultados son significativos por región, por área y por nivel de ocupación. 


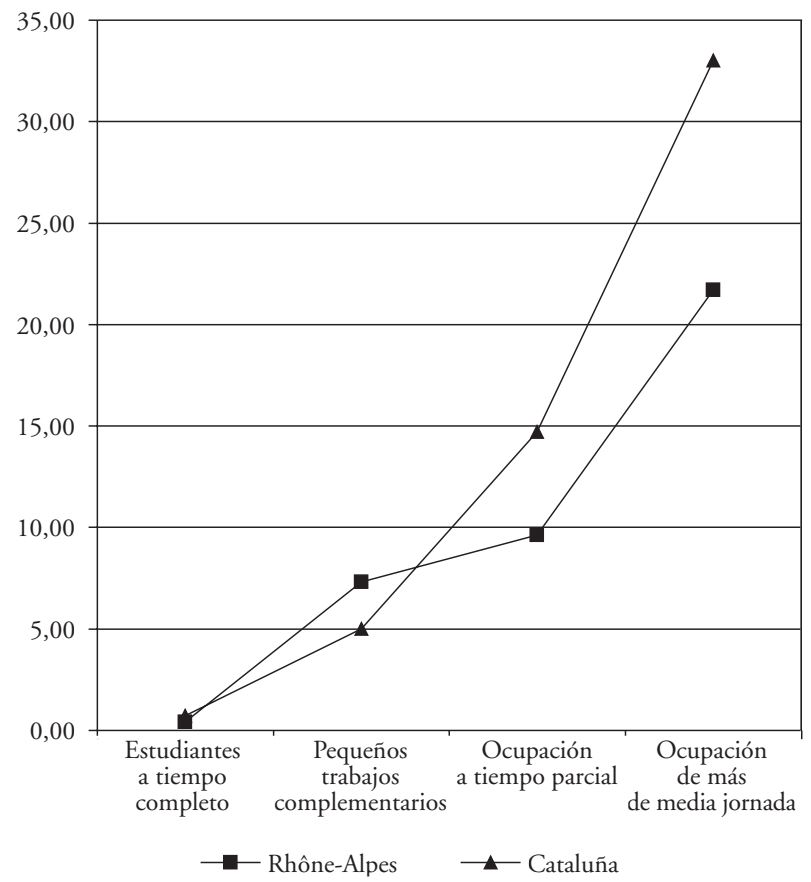

Gráfico 11. Dedicación al trabajo por regiones.

cambio un porcentaje más alto que las otras regiones en «aumentar las oportunidades de inserción» (35\%).

El gráfico $12^{9}$ muestra que hay una relación negativa entre las horas dedicadas al trabajo remunerado y las dedicadas al estudio autónomo sin contar la asistencia a clase, aunque las diferencias no son tan pronunciadas como cabría esperar. Los estudiantes a tiempo completo se dedican al estudio entorno a las 12 horas semanales frente a los estudiantes que trabajan más de media jornada, que sólo dedican entorno a 7,5 horas a la semana. Los datos muestran que las diferencias entre los estudiantes a tiempo completo y los que realizan pequeños trabajos de acompañamiento son muy reducidas.

La introducción del nuevo sistema de créditos europeo (ECTS), que considera como medida fundamental el tiempo total de trabajo de los estudiantes para adquirir los distintos conocimientos y las distintas habilidades, deberá tener en cuenta estas realidades para realizar una planificación adecuada de los planes de estudio.

9. Los resultados de la gráfica se obtienen controlando la variable área de estudio. 


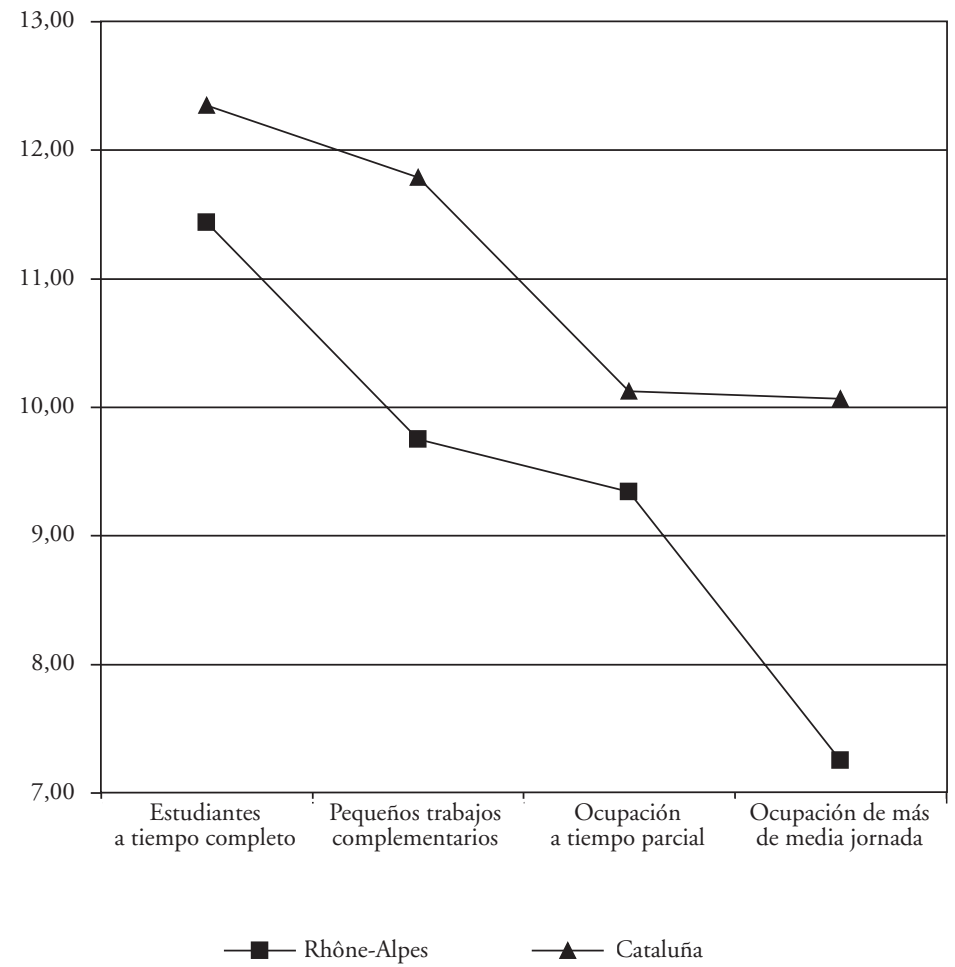

Gráfico 12. Media de horas dedicadas al estudio.

\section{La percepción del éxito en los estudios}

Hemos medido el progreso en los estudios a partir de la percepción subjetiva que los mismos estudiantes muestran sobre el progreso realizado en distintos aspectos relacionados con la actividad académica en la universidad.

Hemos tenido en cuenta una pregunta del cuestionario, transcrita en el apéndice que contiene 14 indicadores. Los encuestados respondieron en cada uno de los indicadores en una escala de 0 a 6 .

Los ítems de la pregunta son los siguientes:

- Conocimientos propios de la titulación.

- Capacidades prácticas, referencias a la profesión y a la práctica.

- Conocimientos fuera del marco de la disciplina e interdisciplinariedad.

- Competencias lingüísticas y expositivas, participación en debates.

- Capacidades intelectuales, espíritu lógico, metódico.

- Capacidades de trabajo en equipo.

- Metodología, técnicas de trabajo y estudio. 


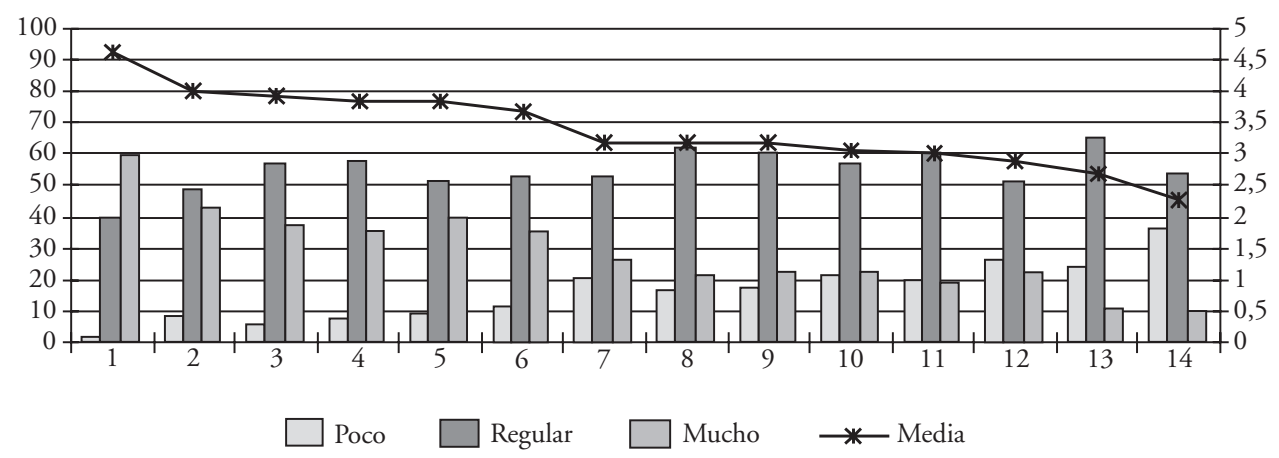

Gráfico 13. Percepción del progreso en los estudios.
1. Contenidos específicos.
8. Metodología.
2. Desarrollo personal.
9. Organización.
3. Resolución problemas.
10. Trabajo en equipo.
4. Capacidades intelectuales.
11. Capacidades prácticas.
5. Autonomía.
12. Responsabilidades soc.
6. Esp. crítico.
13. Interdisciplinariedad.
7. Cultura general.
14. Competencias lingüísticas.

- Capacidad de organización y planificación.

- Cultura general.

- Autonomía e independencia.

- Capacidad de analizar y resolver problemas.

- Espíritu crítico.

- Conciencia de las responsabilidades sociales.

- Desarrollo personal.

El gráfico 13 muestra todos los indicadores ordenados a partir de la media aritmética.

Como puede observarse en el gráfico, superan al 4 de media dos indicadores, en primer lugar, el que se refiere a los conocimientos propios de la titulación y, en segundo lugar, el que se refiere al desarrollo personal. Contrariamente, muestra una puntuación por debajo del aprobado (3) la formación en las responsabilidades sociales, la interdisciplinariedad y las capacidades de expresión. Los demás indicadores se sitúan entorno al aprobado.

A partir de un análisis de componentes principales, hemos extraído 4 factores, que recogen el $63 \%$ de la varianza de los datos, con los cuales proseguimos el estudio (gráficos 14, 15, 16 y 17).

Los cuatro factores son los siguientes:

- Progreso disciplinario: conocimientos, capacidades prácticas, capaciades intelectuales. 
- Progreso interdisciplinario: conocimientos fuera del marco de la disciplina, competencias lingüísticas, capacidades prácticas.

- Progreso metodológico: metodologías de trabajo y estudio, capacidad de organización, capacidad de trabajo en equipo.

- Progreso personal: desarrollo personal, espíritu crítico, cultura general, conciencia de responsabilidades sociales, autonomía, capacidad de resolver problemas.

Es interesante hacer notar que, aún realizando la rotación de los factores, hay dos indicadores muy significativos que correlacionan fuertemente con más de un factor. La capacidad de resolver problemas tiene un peso de 0,49 en el factor que se refiere al progreso personal, 0,46 en el progreso disciplinario y 0,42 en progreso metodológico. El indicador de progreso en habilidades prácticas y profesionales tiene un peso de 0,56 en el factor progreso disciplinario y el 0,55 en progreso interdisciplinario. Se trata, pues, de competencias transversales que los estudiantes perciben que las adquieren de diferentes maneras.

Analizamos a continuación las diferencias por área y región de los cuatro factores indicados.

Es muy interesante darse cuenta de la diferencia de los distintos indicadores que forman este factor. Así, mientras un $60 \%$ de los estudiantes consideran

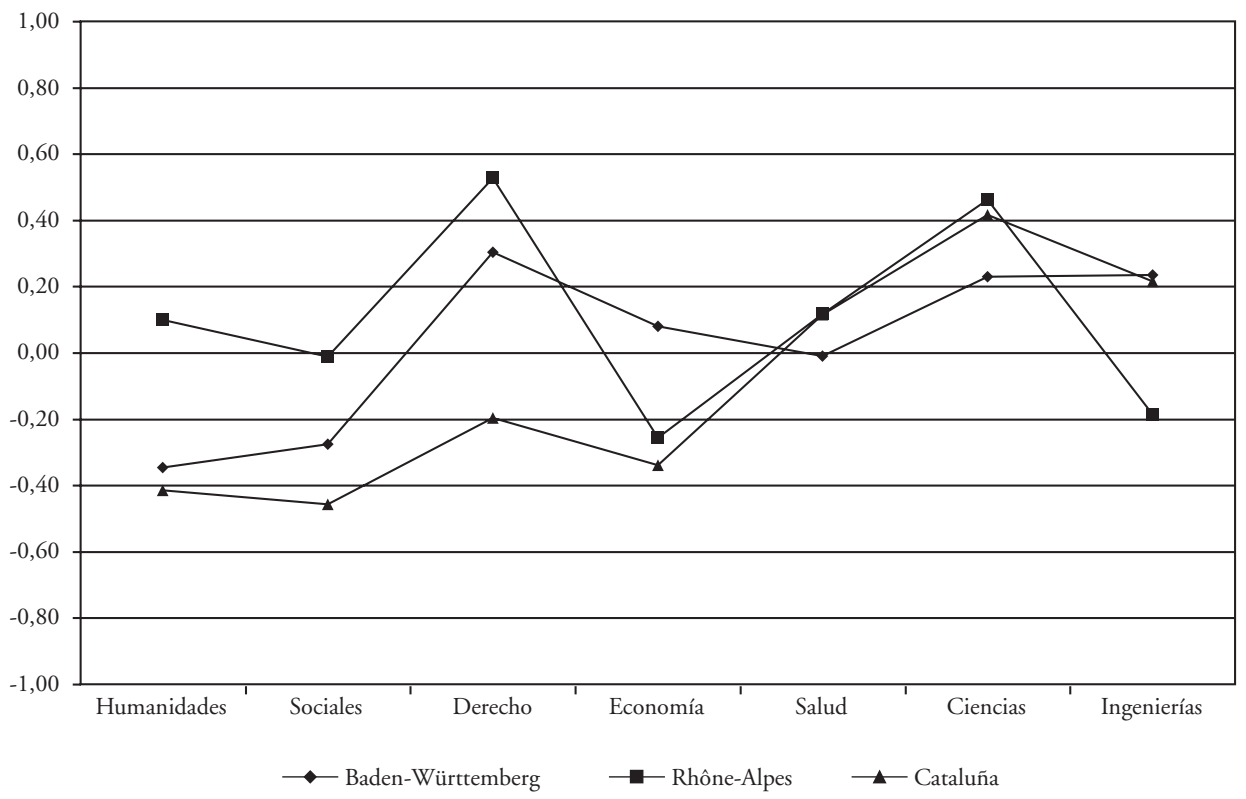

Gráfico 14. Progreso disciplinario. 


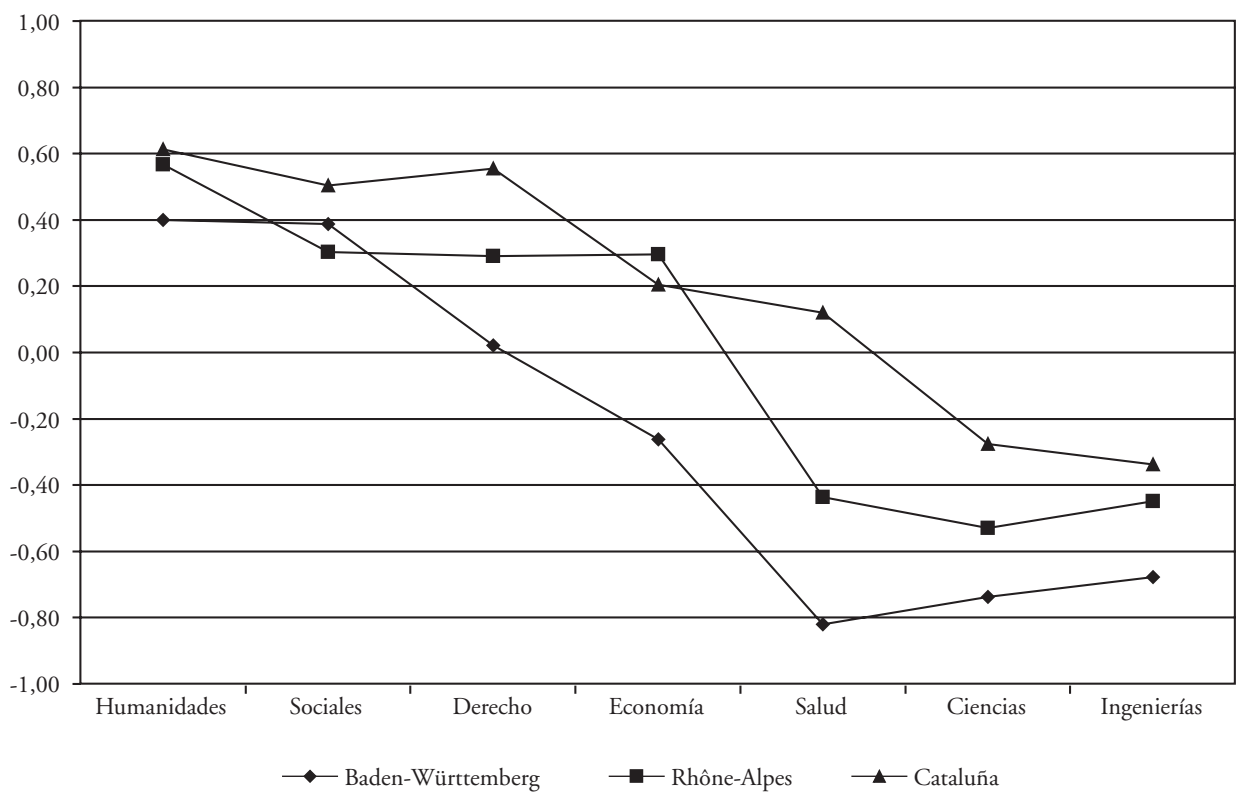

Gráfico 15. Progreso personal.

que progresan mucho en los contenidos de la titulación, sólo un 34\% piensa lo mismo en lo que hace referencia a las capacidades transversales de tipo intelectual como el razonamiento lógico y un $23 \%$ considera que progresa mucho en sus capacidades prácticas o profesionales. Aun que exista esta diferencia, también es significativo que el progreso en algunas habilidades transversales esté asociado con el progreso disciplinario más que con otros indicadores de carácter metodológico o práctico.

La lógica de las áreas es muy clara en este caso. Quedan bien diferenciadas las áreas de Ciencias, Salud e Ingeniería, excepto en Rhône-Alpes, y el área de Derecho en dos de las regiones en comparación con las áreas de Humanidades y Sociales. Como ya hemos visto con anterioridad, el área de Derecho no se comporta igual en Cataluña que en las otras dos regiones, pero esta diferencia no se debe solamente a los conocimientos, ya que sólo destaca la región de Rhône-Alpes, donde un $67 \%$ de los estudiantes considera que adquiere muchos conocimientos frente a un $55 \%$ en las otras dos regiones, sino también a las capacidades intelectuales, donde son los estudiantes alemanes los que valoran más los progresos en este aspecto (39\% frente a 34,5\%).

Cataluña tiende a quedar por debajo de las otras regiones en las áreas de Humanidades, Sociales y Derecho, y en cambio muestra una situación muy semejante en las áreas de Salud, Ciencias e Ingeniería. 


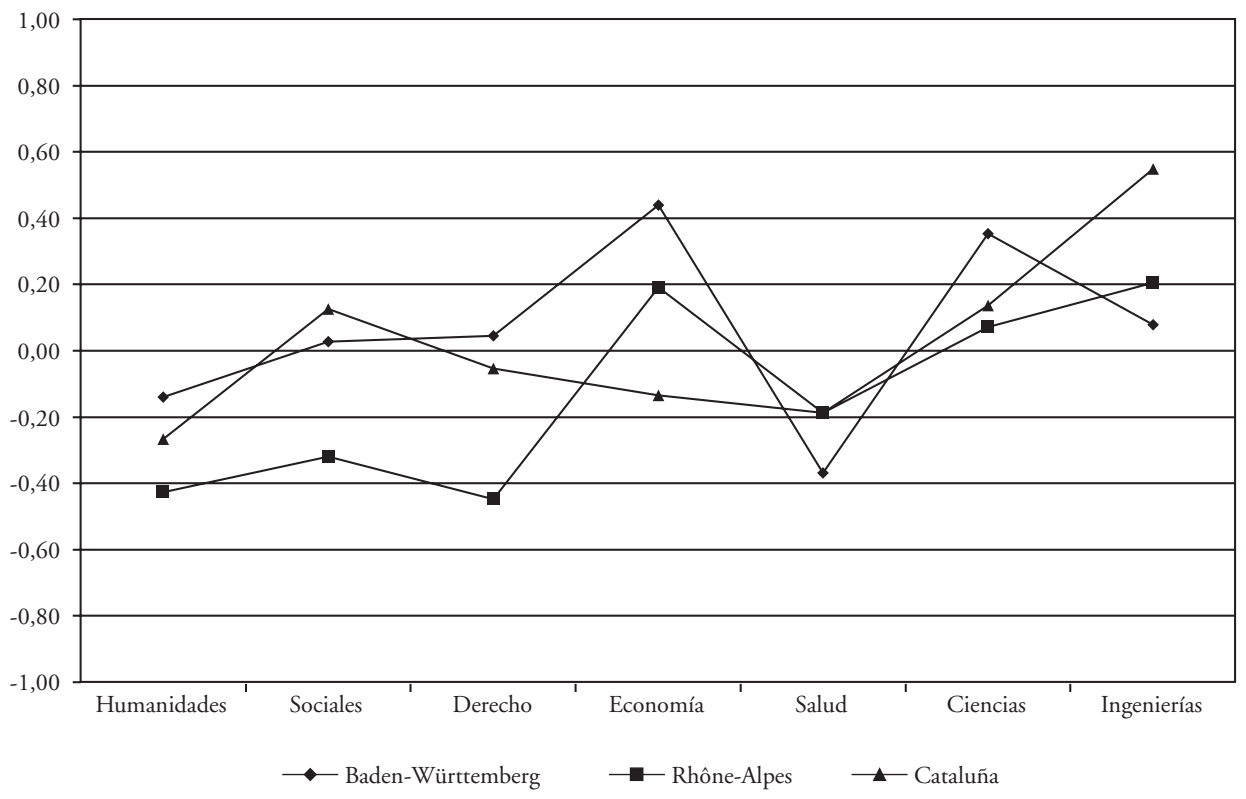

Gráfico 16. Progreso metodológico.

Un 46\% de los encuestados consideran que han progresado mucho en su desarrollo personal, si nos fijamos en el ítem concreto que recoge esta formulación general.

Cataluña es la región que queda mejor situada en general y BadenWürttemberg la que queda peor. La lógica de las titulaciones es bien clara, muestra una clara diferencia entre las áreas de Humanidades y Sociales frente a las áreas de Ciencias, Salud e Ingeniería. Mientras en Cataluña un 50\% de los estudiantes considera que ha progresado en este aspecto, consideran lo mismo el $40 \%$ de los estudiantes de Rhône-Alpes y un 34\% de los de Baden-Württemberg.

Solamente algo más de un $20 \%$ de los encuestados responde que ha progresado mucho en los tres aspectos metodológicos y organizativos que se recogen en este factor.

Las diferencias entre regiones no son claras en esta dimensión, puesto que hay muchas interacciones.

Tampoco es fácil establecer una tendencia clara por titulaciones. En todo caso, sorprende la baja puntuación que el área de la salud obtiene en las tres regiones.

Solamente un $11 \%$ de los encuestados considera que han progresado mucho en los aspectos interdisciplinares y lingüísticos, y un 23\%, tal como indica- 


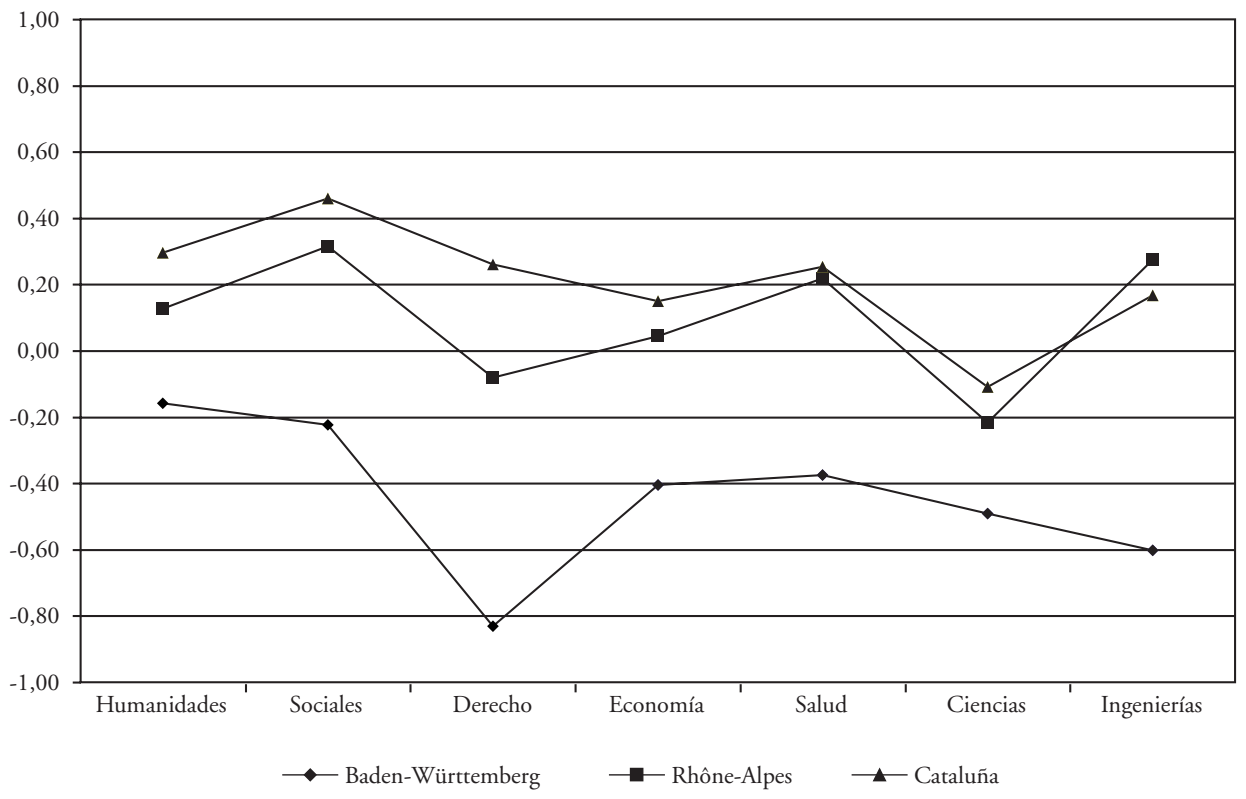

Gráfico 17. Progreso interdisciplinario.

mos anteriormente, en los aspectos prácticos. En general, las áreas de Humanidades, Sociales y Salud quedan mejor situadas.

Cataluña y Rhône-Alpes muestran una situación muy semejante y claramente por encima de la región alemana, donde destaca la baja puntuación relativa de los estudios de Derecho. En concreto, en la región alemana, un $31 \%$ de los encuestados considera que no progresan en interdisciplinariedad frente a un $26 \%$ en Rhône-Alpes y un $19 \%$ en Cataluña. Al mismo tiempo, mientras solamente un $8 \%$ de estudiantes alemanes considera que progresa mucho en capacidades prácticas, responden lo mismo un $22 \%$ de los estudiantes catalanes y un 24\% de los de la región de Rhône-Alpes.

\section{Contraste de hipótesis}

Antes de contrastar las hipótesis centrales de nuestro trabajo anunciadas en la introducción, nos ha parecido relevante relacionar el progreso en los estudios con dos variables que previsiblemente deben influenciar en el progreso en los estudios, por una parte, la aptitud para el estudio medida a partir de las calificaciones de acceso a la universidad y, por otra, la compaginación del estudio con el trabajo remunerado, consideradas dos variables objetivas de las condi- 
ciones del estudio, tal como hemos indicado en la introducción. Éste es, pues, el ejercicio que realizamos en los dos primeros apartados.

¿Existe alguna relación entre las calificaciones de acceso a la universidad y la percepción del progreso en los estudios?

Hemos realizado un análisis de la varianza entre los cuatro factores que se refieren a la percepción del progreso en los estudios y la variable que mide las calificaciones de ingreso en la universidad, manteniendo constantes las áreas de estudio (gráficos 18 y 19). Solamente dos de las cuatro variables dependientes muestran unos resultados significativos, aunque la fuerza de la relación es débil en ambos casos. El progreso disciplinario está positivamente relacionado con la nota de acceso, sobretodo en Baden-Württemberg, y el progreso metodológico también muestra la misma tendencia.

Parece lógico constatar que los estudiantes con mejores notas de acceso sean aquéllos que perciben mejores progresos en aquellos aspectos más académicos de las cuatro variables analizadas, es decir, los aspectos estrictamente

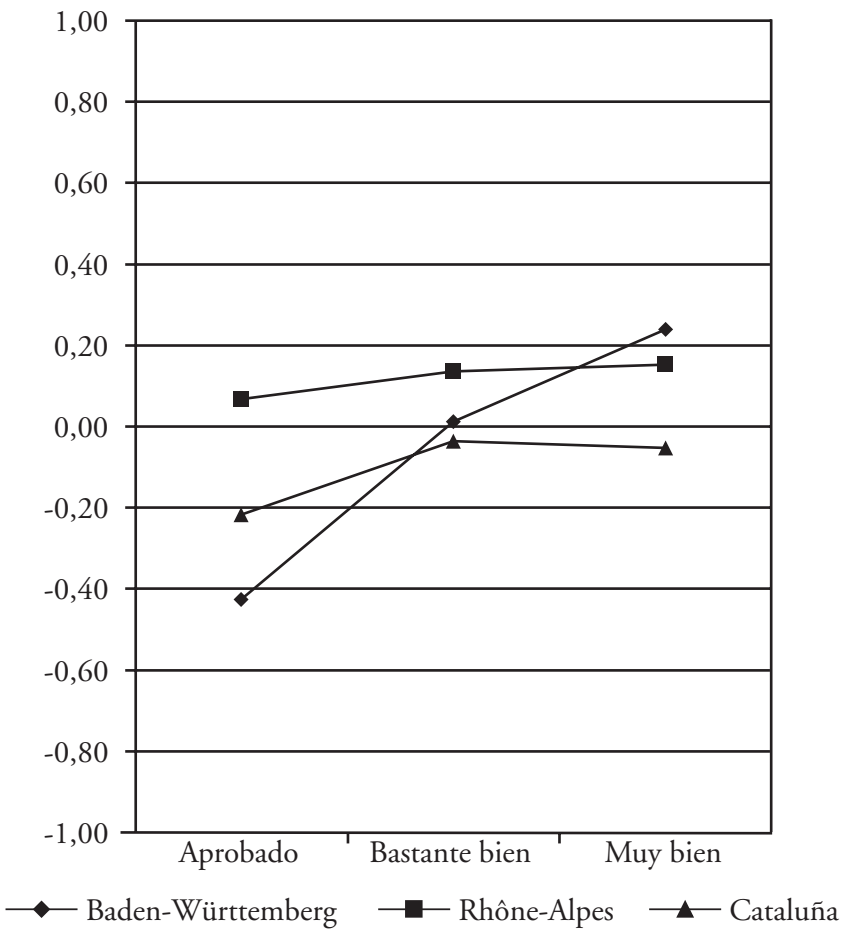

Gráfico 18. Calificaciones de acceso por percepción del progreso disciplinario por región. 


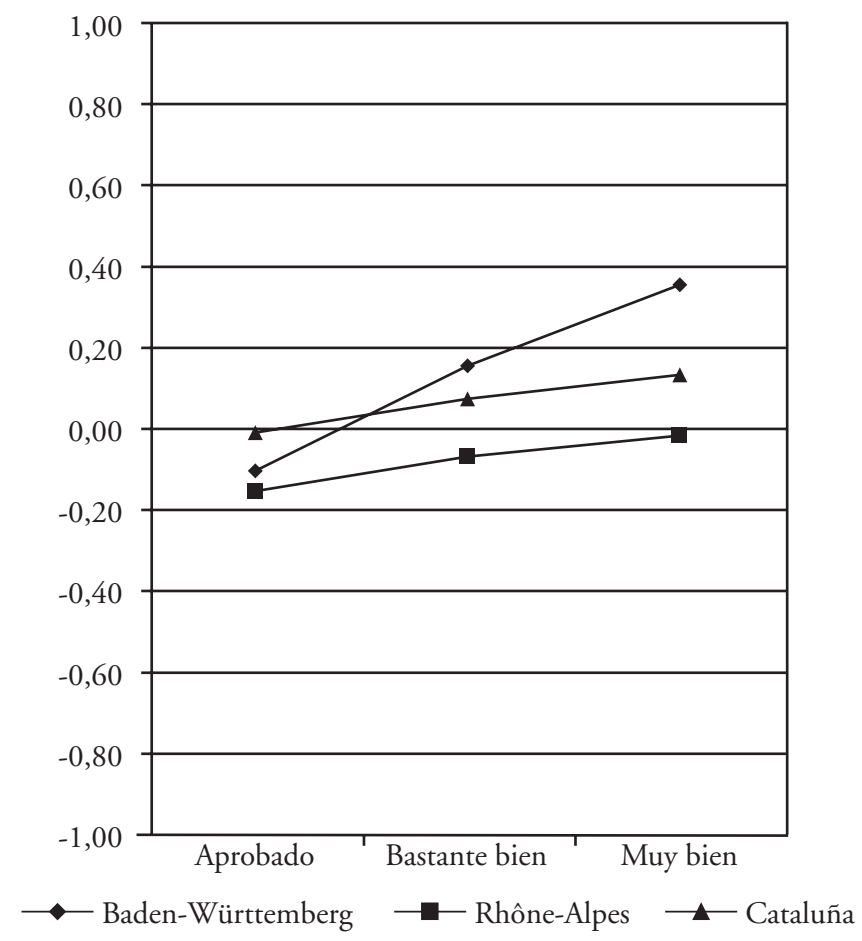

Gráfico 19. Calificaciones de acceso por percepción del progreso metodológico por región.

disciplinares y los metodológicos. De todas maneras, también es interesante constatar que no existe ninguna influencia entre las aptitudes hacia el estudio y la consciencia del progreso en aspectos vinculados con la interdisciplinariedad y la práctica, y con los aspectos que implican un progreso personal, puesto que sugiere que ambos aspectos son más independientes de las aptitudes y dependen más de la experiencia universitaria en un sentido más amplio y de las oportunidades de tener experiencias prácticas asociadas con los estudios.

¿Existe alguna relación entre la participación en el mercado laboral y el progreso en los estudios?

Con el mismo procedimiento metodológico, hemos analizado la relación entre la participación en el mercado laboral y la percepción del progreso en los estudios con las cuatro dimensiones factoriales analizadas.

Solamente el progreso práctico muestra una pequeña diferencia significativa favorable a los estudiantes que trabajan más de media jornada, como muestra el gráfico 20 . 


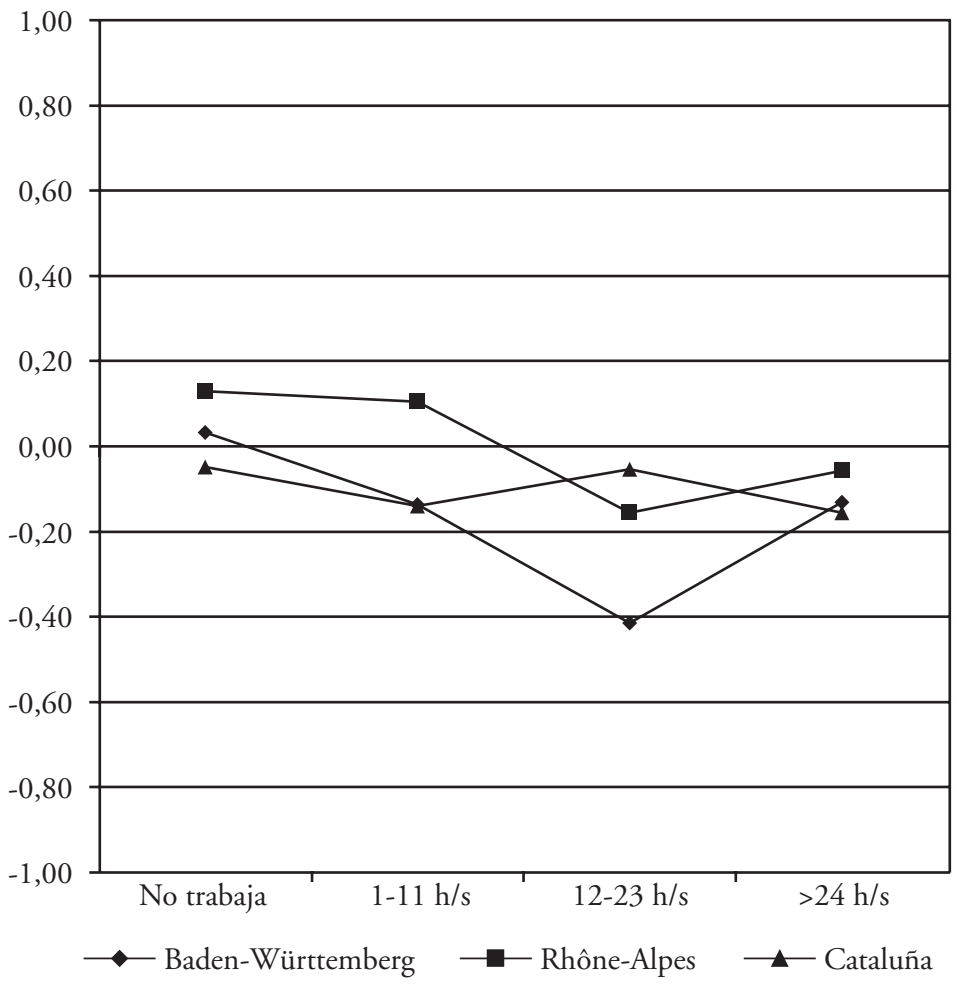

Gráfico 20. Participación en el mercado laboral por progreso práctico por regiones.

No deja de ser sorprendente que en Rhône-Alpes y en Cataluña los estudiantes que trabajan no perciban más dificultades para progresar que el resto en los aspectos disciplinarios, metodológicos y personales. En todo caso, aquéllos que trabajan más de media jornada perciben un progreso interdisciplinario y práctico algo superior que el resto, lo cual puede ser indicador de una buena relación entre los estudios y el trabajo que realizan.

¿Cuál es la relación entre la percepción de las condiciones de estudio, las motivaciones intrínsecas y extrinsecas y la percepción del progreso?

Para contrastar la hipótesis central de nuestra investigación, hemos realizado un modelo de regresión lineal entre cada uno de los cuatro factores de progreso, las dos variables motivacionales y las tres variables que miden las condiciones en el estudio. Los resultados se expresan sintéticamente en la tabla 1. Con posterioridad, hemos comprobado si los resultados obtenidos se mante- 
Tabla 1. Relación entre percepciones de condiciones de estudio, motivaciones y progreso.

\begin{tabular}{lllll}
\hline & P. personal & P. disciplinario & P. metodológico & P. interdisciplinario \\
\hline $\begin{array}{l}\text { R2 en distintos } \\
\text { modelos }\end{array}$ & $0,12,0,13$, & $0,24,0,30$, & $0,25,0,29$, & $0,31,0,31$, \\
\hline M. expresiva & $0,14,0,15$ & $0,34,0,36$ & $0,31,0,31$ & 0,32 \\
\hline M. instrumental & $0,04^{*}$ & $0,15^{*}$ & - & - \\
\hline O. profesional & $-0,06^{*}$ & - & $0,13^{*}$ & - \\
\hline $\begin{array}{l}\text { O. especializada } \\
\text { Estudios con }\end{array}$ & $-0,12^{*}$ & $0,12^{*}$ & $0,24^{*}$ & $0,31^{*}$ \\
\hline desventajas & - & $0,22^{*}$ & $0,09^{*}$ & $-0,05^{*}$ \\
\hline
\end{tabular}

1. Esta fila indica los distintos valores del R2 a medida que se van introduciendo las distintas variables.

nían para los distintos valores de las variables analizadas en los dos apartados anteriores, para asegurarnos que no se trata de efectos espúreos.

Los datos muestran coeficientes de correlación débiles, aunque estadísticamente significativos y en la dirección de la hipótesis planteada al inicio del análisis.

La motivación expresiva tiene que ver con la percepción del progreso personal y disciplinario. Así pues, en aquellas titulaciones orientadas disciplinarmente y con mayor exigencia, se requiere para el éxito una implicación mayor de los alumnos. Parece razonable igualmente que los alumnos a los que menos les gusta estudiar se sientan menos adaptados a la universidad y por consiguiente consideren que menos progresan personalmente.

Los alumnos perciben un mayor progreso metodológico e interdisciplinario cuando tienen conciencia que los estudios están más orientados hacia la profesionalidad y la práctica.

Los estudiantes motivados instrumentalmente perciben mejor su progreso metodológico, que implica, como hemos visto, aspectos de organización.

Los estudios que son percibidos como más especializados y exigentes se traducen en una percepción de mayor progreso en esta dirección.

Los estudiantes con un nivel más alto de motivaciones instrumentales son los que perciben más negativamente su progreso personal. Lo mismo sucede cuando los estudios tienden a tener una orientación especializada y exigente.

Los resultados anteriores se mantienen tanto entre los estudiantes con notas de ingreso altas como bajas, igualmente entre los estudiantes a tiempo completo y los que trabajan más de media jornada, con lo cual podemos afirmar que no se deben a relaciones encubiertas producidas por las diferentes aptitudes hacia los estudios o la diferente participación en el mercado laboral. 


\section{¿Existe alguna relación entre el sexo y el origen social con las motivaciones instrumentales y expresivas de los estudiantes?}

La contrastación de la segunda hipótesis que nos hemos planteado ofrece unos resultados interesantes porque se apartan en algún aspecto de las expectativas teóricas.

$\mathrm{Ni}$ el origen social ni el sexo muestran una relación significativa con las motivaciones expresivas o instrumentales, si mantenemos constantes las áreas de estudio. El filtro que supone la entrada en la universidad anula la importancia que la personalidad, en el caso del género, y la socialización primaria, en ambos casos, pudiera tener en las motivaciones.

\section{Discusión de los resultados y conclusiones}

1. La descripción de las motivaciones de los estudiantes nos muestra las diferencias entre las distintas áreas de estudio, las cuales siguen una lógica semejante en las tres regiones estudiadas. Aunque las tradiciones de cada país puedan ser diferentes, cuando un joven elige una carrera de ciencias o una de económicas, para poner un ejemplo, sus preferencias y expectativas en relación con la universidad son significativamente distintas i relativamente semejantes entre países.

La diferencia más importante entre las áreas se establece a partir de las motivaciones instrumentales, lo cual indica que los estudiantes que eligen determinados estudios como económicas, derecho e ingeniería perciben que sus posibilidades y expectativas de inserción y de promoción son superiores. Esta realidad no puede oscurecer que los motivos predominantes en todos los casos son los expresivos, es decir, la elección de una determinada titulación a partir del interés por la misma. En el extremo de la importancia atribuida a los valores expresivos, encontramos que uno de cada diez estudiantes no muestra ninguna preocupación por su futuro profesional.

Los resultados constatan la diferencia entre los estudiantes sobretodo entre áreas de estudio y ofrecen un instrumento de reflexión a la hora de diseñar la programación y la práctica de los estudios en orden a ofrecer elementos para compensar o reforzar las tendencias apuntadas.

2. La profesionalización de los estudios universitarios ha sido una de las políticas impulsada en Europa en las últimas décadas, sin embargo, hemos observado que predominan los estudiantes que perciben los estudios elegidos como más orientados disciplinariamente que no hacia la práctica de una profesión. Posiblemente, aunque el discurso pedagógico oficial se orienta hacia la profesionalización, puede ser que en algunas universidades europeas no se haya traducido en reformas de los planes de estudio o bien que el profesorado universitario, a la hora de aplicar las reformas en la práctica cotidiana, las recontextualiza según su propia tradición, experiencia y recur- 
sos. Este aspecto señalado ampliamente por Berstein (1990) debería servir de alerta para todos los que creen erróneamente que, una vez diseñada una reforma y traducida en normas, empieza a tener efectos en la práctica social.

Un aspecto a destacar es que los estudios de derecho, cuya tradición profesional es indiscutible, muestran una orientación profesionalizadora y práctica muy débil, a juicio de los estudiantes, sobretodo en dos de las tres regiones. Las causas de este resultado, aparentemente contradictorio, pueden deberse precisamente a efectos perversos relacionados con su larga tradición profesional y a la presencia de colegios profesionales con una fuerte capacidad de control e influencia. Esta realidad institucional histórica se ha traducido en unos estudios universitarios orientados académicamente, más bien rígidos y unitarios, a la espera que una vez terminados los mismos se acceda a la profesión de abogado a partir de la especialización organizada por parte de los colegios o bien a través de la práctica profesional en despachos de profesionales (los pasantes).

Se trata de una realidad poco adaptada a la situación actual, en la cual los estudios de derecho son utilizados por un amplio sector de graduados como canal de acceso a un abanico de actividades profesionales en el campo de la administración y la gestión muy distintos al ejercicio de la abogacía.

Los estudios del área de Salud, también con una gran tradición profesional, son percibidos por parte de los estudiantes al mismo tiempo como profesionalizadores, prácticos y especializados. Se trata de una percepción sustentada sobre la realidad de la presencia ininterrumpida de experimentación y de práctica clínica en hospitales durante los estudios y de una orientación centrada en la especialidad médica, veterinaria o farmacéutica, según los $\operatorname{casos}^{10}$.

3. En las tres regiones estudiadas, la presencia de titulaciones en desventaja relativa debidas a problemas de masificación y exceso de competitividad no parece que sea un problema que afecte a muchos estudiantes. En todo caso, los principales problemas en este sentido se presentan en Cataluña.

4. La compaginación de los estudios con el trabajo remunerado entre los estudiantes que teóricamente frecuentan la universidad como alumnos presenciales es una práctica importante en las tres regiones, pero sobretodo en Cataluña. Las dificultades en el mercado laboral están entre las causas que influyen en la tendencia a buscar una inserción profesional previa, que facilite la definitiva una vez terminados los estudios, sobretodo en Cataluña, donde las razones relacionadas con la financiación de los estudios son menos importantes.

10. Cabe indicar que las titulaciones son diferentes en las tres regiones, lo cual introduce confusión en los resultados. En la región alemana se incluyen únicamente estudiantes de Medicina, en la francesa, estudiantes de Medicina y de Farmacia, y en la catalana, de Medicina y Veterinaria. 
Es importante resaltar la importancia atribuida por un número cercano a la mitad de los estudiantes que trabajan a la necesidad de financiar las propias necesidades de ocio. La realización de estudios largos y en algunos casos las dificultades de inserción se traducen en un retraso en el abandono de la familia de procedencia, pero también en la búsqueda de recursos, por lo menos para satisfacer las propias necesidades de consumo.

5. El análisis de la percepción por parte de los estudiantes del progreso que realizan en la universidad reafirma lo que hemos indicado anteriormente en el apartado sobre profesionalización, ya que es muy superior el progreso disciplinario que el progreso en competencias transversales indispensables para el ejercicio de una profesión y en la incorporación de la interdisciplinariedad en las titulaciones, problema que parece plantearse con especial crudeza en la región alemana.

Los datos también muestran la clara diferencia entre la cultura de ciencias y la cultura de las humanidades, en el sentido que los estudiantes de las áreas de Ciencias manifiestan que han progresado menos a nivel personal que los de las áreas más humanísticas. Particularmente relevante es el caso de Medicina, si se tiene en cuenta que se trata de una profesión cuyo ejercicio tiene un claro componente humano.

6. Los resultados obtenidos confirman en general la hipótesis de partida de que la percepción del progreso en la universidad está condicionada por la motivación y las condiciones de las titulaciones. De todas maneras, los resultados no son espectaculares y muestran que otras variables son también muy importantes y que los indicadores utilizados no acaban de captar el fenómeno que se pretende medir. Se trata de fenómenos subjetivos, puesto que se refieren a percepciones de realidades objetivas como la organización de los estudios sobre las cuales los estudiantes no poseen elementos suficientes de contraste y de fenómenos totalmente subjetivos, como la percepción del propio progreso en diferentes aspectos relacionados con la vida universitaria. Se trata, pues, de aspectos muy complejos que no se dejan abarcar fácilmente con las metodologías utilizadas y que se ven afectados por el fenómeno de la reflexividad. Es decir, puede ser que los estudiantes perciban de una manera determinada la organización de los estudios precisamente porque consideran que han progresado en esta dirección, lo que crea un círculo vicioso en la contrastación de la hipótesis.

Prescindiendo del problema metodológico indicado, existen algunos hallazgos que pueden orientar políticas de implementación de reformas en la universidad. Hemos podido confirmar que cuando las titulaciones están más orientadas hacia la profesionalidad, los estudiantes perciben que su progreso en los aspectos interdisciplinares, metodológicos y personales es superior. En cambio, cuando las titulaciones están muy orientadas disciplinariamente, el progreso personal y el interdisciplinario es negativo. Es 
decir que existe cierta coherencia entre los propósitos de las reformas y la percepción de los estudiantes de su propio progreso.

Aunque la relación es muy débil, también podemos constatar que para tener éxito en una titulación muy orientada disciplinariamente que además suele caracterizarse por la exigencia, es importante una motivación expresiva alta hacia la disciplina elegida, lo cual forma parte de la cultura académica de sentido común, pero sugiere la necesidad de orientar a los candidatos en estos aspectos antes de que realicen sus propias opciones a través de la matrícula, puesto que tienen un alto riesgo de fracaso si no están muy motivados.

7. Tal como se ha indicado anteriormente, la compaginación de los estudios con el trabajo remunerado no ejerce demasiada influencia en la percepción que los estudiantes tienen de sus propios progresos, sobretodo de los progresos en las dimensiones más aplicadas de los estudios, sin embargo, nos consta, a partir de los datos de esta investigación, que tiene relación con el tiempo que los estudiantes emplean para obtener el correspondiente diploma, lo cual hace pensar que las universidades deberían tener en cuenta esta realidad a la hora de planificar los estudios.

8. La segunda hipótesis de nuestro estudio ha demostrado ser falsa. Por una parte, no existe ninguna relación entre el origen social y las motivaciones hacia los estudios, lo cual refuerza la idea que los condicionantes de origen social de la socialización primaria han sido debilitados por los cambios en la estructura social, la influencia de la enseñanza media y de la cultura mediática actual. La parte de los estudiantes que consiguen un cierto éxito académico en la escuela secundaria, de manera que pueden orientarse hacia la universidad con más o menos interés, organizan sus preferencias independientemente de los códigos asociados a su pertenencia de clase social.

Por otra parte, las mujeres, aunque en su socialización primaria no hayan sido orientadas hacia los estudios y la profesión, tienen experiencia en la escuela secundaria de su capacidad para los mismos, en muchos casos superior a los de sus compañeros varones, y perciben la necesidad instrumental de estudiar para conseguir más autonomía en la vida familiar y social, lo cual les hace muy semejantes, en cada área de estudio, a sus compañeros varones en lo que a motivaciones se refiere.

En definitiva, por una parte, los cambios en la estructura de clases, en la participación de las mujeres en la sociedad y en los valores sociales compartidos y, por otra, el hecho de que los jóvenes y sobretodo las jóvenes hayan aumentado mucho su presencia en la universidad pero después de un proceso de selección fundamentalmente académica, cuestiona la teoría que relaciona la socialización primaria en la familia con la socialización secundaria en el sistema educativo cuando se trata de la educación superior. 


\section{Apéndice}

\section{Motivaciones}

Para medir las motivaciones de los estudiantes, hemos utilizado una pregunta del cuestionario con siete ítems que han sido reducidos mediante un análisis factorial fijado en tres factores, de acuerdo con la propuesta teórica, que en conjunto resumen el $71 \%$ de la varianza de los datos. Las tablas 1,2 y 3 resumen los parámetros más importantes asociados a cada factor.

Tabla 1. Motivación instrumental.

\begin{tabular}{lcccccc}
\hline & Media & Desv. t. & Mucho & Reg. & Poco & Peso \\
\hline $\begin{array}{l}\text { Expectativas de buenos } \\
\text { ingresos en una profesión }\end{array}$ & 3,2 & 1,8 & 25 & 55 & 21 & 0,82 \\
$\begin{array}{l}\text { futura } \\
\begin{array}{l}\text { Diversidad de oportunidades } \\
\text { profesionales }\end{array}\end{array}$ & 3,8 & 1,8 & 41 & 46 & 13 & 0,75 \\
\hline $\begin{array}{l}\text { Perspectivas de conseguir } \\
\text { una ocupación segura }\end{array}$ & 3,3 & 1,9 & 31 & 48 & 21 & 0,79 \\
\hline $\begin{array}{l}\text { Buenas perspectivas de llegar } \\
\text { a ser un ejecutivo en el futuro }\end{array}$ & 2,3 & 1,9 & 17 & 41 & 42 & 0,81 \\
\hline
\end{tabular}

Tabla 2. Motivación expresiva.

\begin{tabular}{lcccccc}
\hline & Media & Desv. t. & Mucho & Reg. & Poco & Peso \\
\hline $\begin{array}{l}\text { Interés específico } \\
\text { por la materia }\end{array}$ & 5 & 1,2 & 72 & 27 & 1 & 0,74 \\
\hline $\begin{array}{l}\text { Aptitudes personales, } \\
\text { capacidades }\end{array}$ & 4,3 & 1,2 & 46 & 52 & 2 & 0,88 \\
\hline
\end{tabular}

Tabla 3. Motivación profesional.

\begin{tabular}{lcccccc}
\hline & Media & Desv. t. & Mucho & Reg. & Poco & Peso \\
\hline $\begin{array}{l}\text { Una opción estrechamente } \\
\text { relacionada con } \\
\text { una profesión }\end{array}$ & 3,3 & 1,9 & 31 & 48 & 21 & $-0,75$ \\
\hline
\end{tabular}

\section{Características de las titulaciones}

Para medir la percepción de los estudiantes sobre las características de las titulaciones, hemos utilizado una pregunta del cuestionario con once ítems que ha sido reducida mediante un análisis factorial, del cual han salido tres factores con un valor propio mayor que uno que resumen el $49 \%$ de la varianza de 
los datos. Las tablas 4, 5 y 6 resumen los parámetros más importantes asociados a cada factor.

Tabla 4. Estudios profesionalizados y prácticos

\begin{tabular}{lcccccc}
\hline & Media & Desv. t. & Mucho & Reg. & Poco & Peso \\
\hline $\begin{array}{l}\text { Buena preparación para } \\
\text { la profesión, estrecha relación } \\
\text { con la práctica }\end{array}$ & 2,2 & 1,6 & 9 & 53 & 38 & 0,72 \\
\hline $\begin{array}{l}\text { Buenas relaciones entre } \\
\text { estudiantes y docentes }\end{array}$ & 3,3 & 1,5 & 23 & 66 & 12 & 0,66 \\
$\begin{array}{l}\text { Introducción de nuevos } \\
\text { medios didácticos. }\end{array}$ & 2,4 & 1,6 & 13 & 55 & 33 & 0,69 \\
\hline $\begin{array}{l}\text { Relación entre la enseñanza } \\
\text { y la investigación }\end{array}$ & 2,8 & 1,6 & 15 & 63 & 23 & 0,59 \\
\hline $\begin{array}{l}\text { Claridad en las exigencias } \\
\text { de los exámenes }\end{array}$ & 3,1 & 1,4 & 17 & 68 & 14 & 0,53 \\
\hline
\end{tabular}

Tabla 5. Estudios especializados y exigentes

\begin{tabular}{lllllrl}
\hline & Media & Desv. t. & Mucho & Reg. & Poco & Peso \\
\hline Orientación especializada & 3,3 & 1,6 & 23 & 63 & 14 & 0,56 \\
\hline Mucha exigencia & 4,0 & 1,4 & 39 & 56 & 5 & 0,82 \\
\hline Estudios bien estructurados & 3,2 & 1,4 & 18 & 70 & 12 & 0,56 \\
\hline
\end{tabular}

Tabla 6. Estudios masificados en desventaja

\begin{tabular}{lllllll}
\hline & Media & Desv. t. & Mucho & Reg. & Poco & Peso \\
\hline $\begin{array}{l}\text { Estudiantes en desventaja } \\
\text { en relación con otras titulaciones }\end{array}$ & 1,6 & 1,8 & 10 & 33 & 57 & 0,69 \\
\hline Cursos con demasiados estudiantes & 2,3 & 1,9 & 17 & 42 & 42 & 0,68 \\
\hline Competitividad entre estudiantes & 2,4 & 1,8 & 16 & 47 & 37 & 0,60 \\
\hline
\end{tabular}

\section{Percepción del progreso en los estudios}

Para medir la percepción del progreso realizado en la universidad, hemos utilizado una pregunta del cuestionario con 14 indicadores. A partir de un análisis factorial, hemos reducido la información a cuatro factores (valor propio mayor que 1) que resumen el 63\% de la varianza. Las tablas 7, 8, 9 y 10 resumen la relación entre ejes e indicadores. 
Tabla 7. Progreso personal.

\begin{tabular}{lllllll}
\hline & Media & Desv. & Mucho & Reg. & Poco & Peso \\
\hline Desarrollo personal & 4,0 & 1,5 & 43 & 49 & 8 & 0,74 \\
\hline Espíritu crítico & 3,7 & 1,6 & 36 & 53 & 12 & 0,72 \\
\hline Cultura general & 3,2 & 1,8 & 26 & 53 & 20 & 0,67 \\
\hline $\begin{array}{l}\text { Conciencia responsabilidades } \\
\text { sociales }\end{array}$ & 2,9 & 1,8 & 23 & 51 & 27 & 0,66 \\
\hline Autonomía e independencia & 3,9 & 1,6 & 40 & 51 & 10 & 0,64 \\
\hline Capacidad de resolver problemas & 3,9 & 1,4 & 37 & 57 & 6 & 0,50 \\
\hline
\end{tabular}

Tabla 8. Progreso metodológico.

\begin{tabular}{lcccccc}
\hline & Media & Desv. & Mucho & Reg. & Poco & Peso \\
\hline $\begin{array}{l}\text { Metodología, técnicas de trabajo } \\
\text { y de estudio }\end{array}$ & 3,2 & 1,6 & 22 & 62 & 17 & 0,82 \\
\hline $\begin{array}{l}\text { Capacidad de organización } \\
\text { y planificación }\end{array}$ & 3,2 & 1,6 & 22 & 60 & 18 & 0,81 \\
\hline Capacidad de trabajar en equipo & 3,1 & 1,7 & 22 & 57 & 21 & 0,72 \\
\hline
\end{tabular}

Tabla 9. Progreso interdisciplinario

\begin{tabular}{lcccccc}
\hline & Media & Desv. & Mucho & Reg. & Poco & Peso \\
\hline $\begin{array}{l}\text { Conocimientos fuera del marco } \\
\text { de la disciplina } \\
\text { e interdisciplinariedad }\end{array}$ & 2,7 & 1,5 & 11 & 66 & 24 & 0,78 \\
$\begin{array}{l}\text { Competencias lingüísticas, } \\
\begin{array}{l}\text { expositivas y participación } \\
\text { en discusiones }\end{array}\end{array}$ & 2,3 & 1,6 & 10 & 54 & 36 & 0,69 \\
\hline $\begin{array}{l}\text { Capacidades prácticas, referencia } \\
\text { a la profesión y a la práctica }\end{array}$ & 3,0 & 1,6 & 19 & 60 & 20 & 0,55 \\
\hline
\end{tabular}

Tabla 10. Progreso disciplinario.

\begin{tabular}{lcccccc}
\hline & Media & Desv. & Mucho & Reg. & Poco & Peso \\
\hline $\begin{array}{l}\text { Conocimientos propios } \\
\text { de la titulación }\end{array}$ & 4,6 & 1,2 & 59 & 39 & 2 & 0,84 \\
\hline $\begin{array}{l}\text { Capacidades prácticas, referencia } \\
\text { a la profesión y a la práctica }\end{array}$ & 3,0 & 1,6 & 19 & 61 & 20 & 0,56 \\
\hline $\begin{array}{l}\text { Capacidades intelectuales } \\
\text { (espíritu lógico, metódico) }\end{array}$ & 3,9 & 1,4 & 35 & 58 & 7 & 0,45 \\
\hline Capacidad de resolver problemas & 3,9 & 1,4 & 37 & 57 & 6 & 0,46 \\
\hline
\end{tabular}


Tabla 11. Distribución de hombres y mujeres por grupos de disciplinas y por región (en porcentaje).

\begin{tabular}{|c|c|c|c|c|c|c|c|c|c|c|}
\hline & & Humanidades & Sociales & Derecho & Economía & Salud & Ciencias & Ingenierías & Otras & Total \\
\hline \multirow[t]{3}{*}{ Baden-Württemberg } & Hombres & 33,1 & 32,7 & 50,9 & 73,8 & 39,4 & 65,8 & 85,7 & 49,2 & 58,2 \\
\hline & Mujeres & 66,9 & 67,3 & 49,1 & 26,2 & 60,6 & 34,2 & 14,3 & 50,8 & 41,8 \\
\hline & Total & 100 & 100 & 100 & 100 & 100 & 100 & 100 & 100 & 100 \\
\hline \multirow[t]{3}{*}{ Rhône-Alpes } & Hombres & 15,5 & 15,3 & 24,7 & 53,9 & 23,8 & 46,4 & 91,2 & & 37,6 \\
\hline & Mujeres & 84,5 & 84,7 & 75,3 & 46,1 & 76,2 & 53,6 & 8,8 & & 62,4 \\
\hline & Total & 100 & 100 & 100 & 100 & 100 & 100 & 100 & & 100 \\
\hline \multirow[t]{3}{*}{ Cataluña } & Hombres & 27,9 & 21,0 & 22,2 & 45,3 & 22,8 & 47,5 & 79,2 & & 39,0 \\
\hline & Mujeres & 72,1 & 79,0 & 77,8 & 54,7 & 77,2 & 52,5 & 20,8 & & 61,0 \\
\hline & Total & 100 & 100 & 100 & 100 & 100 & 100 & 100 & & 100 \\
\hline
\end{tabular}


Tabla 12. Distribución de los estudiantes según la profesión del padre, el área y la región (en porcentaje).

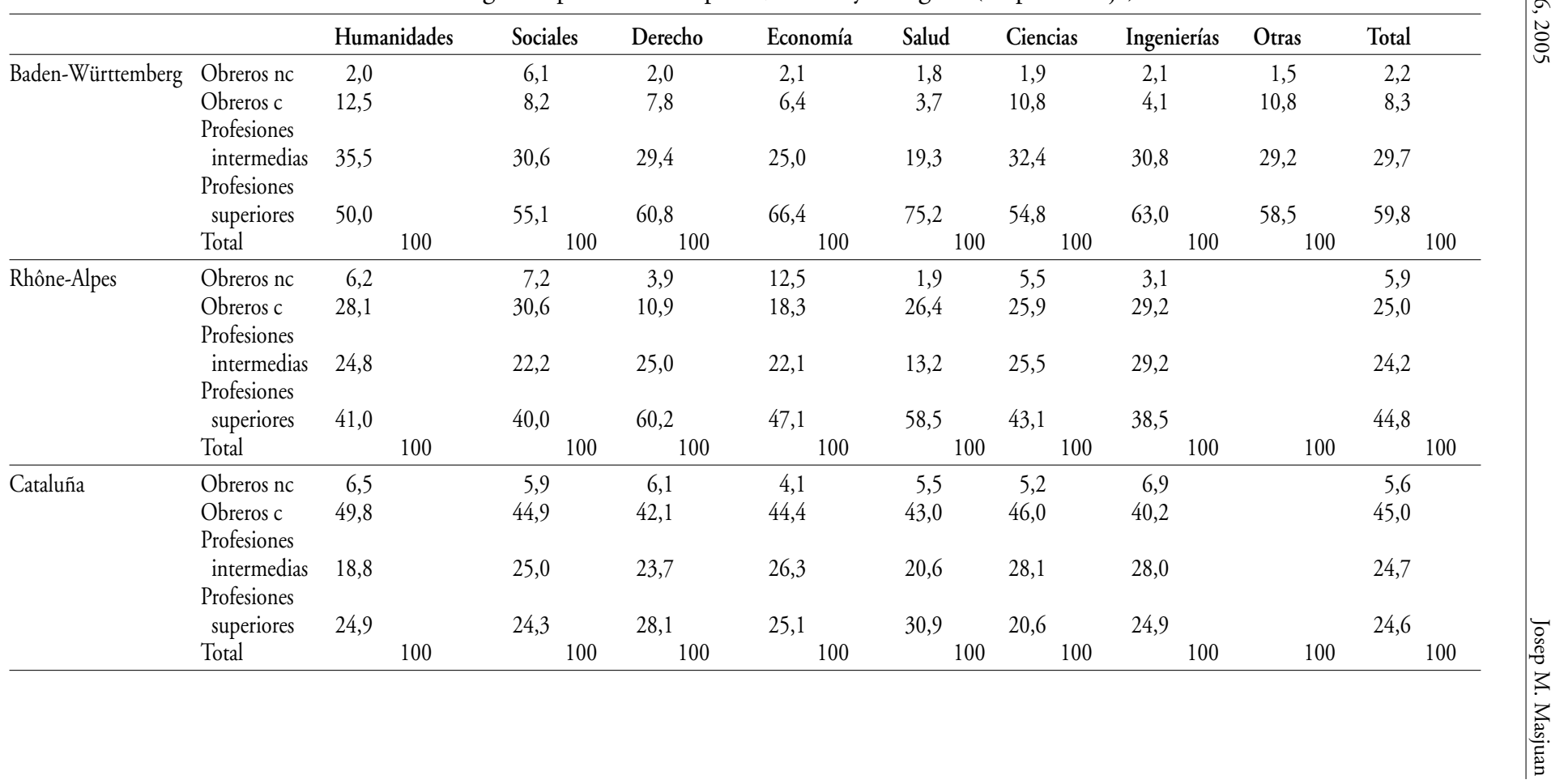




\section{Bibliografía}

BarnetT, R. (2000). "Supercomplexity and the Curriculum». Studies in Higher Education, vol. 25(3), p. 255-265.

Brown, P.; SCASE, R. (1994). Higher Education and Corporate Realities. Class, Culture and Decline of Graduate Careers. Londres: UCL Press.

BeduWÉ, C.; Planas, J. (2003). Educational Expansion and Labour Marked. Thessaloniki: Cedefop.

Berstein, B. (1990). La estructura del discurso pedagógico. Madrid: Morata.

Bourdieu, P.; PASSERON, J.C. (1969). Los estudiantes y la cultura. Barcelona: Labor.

CRYER, P.; ELTON, L. (1990). "Catastrophe Theory: a unified model for educational Change». Studies in Higher Education, 15-1, p. 75-87.

FERnÁndeZ EnGuita, M. (1988). «Yo no soy esto que tú te imaginas». Cuadernos de Pedagogía.

HadjI, Ch.; FerneX, A.; Lima, L. (2003). Les étudiants de la Région Rhône-Alpes face a leurs études. LSE, UPMF Grenoble (edición policopiada).

Herberg, F.; Mauser, B.; Snyderman, B. (1959). The Motivation to Work. Nueva York: Willey.

Johnson, R.N.; DeEM, R. (2003). «Talking of Students: Tensions and contradictions for the manager-academic and the university in comptemporary higher education». Higher Education, 46, p. 289-314.

Masjuan, J.M.; Troiano, H.; ViVAS, J. (2002). I després de la universitat, què? La inserció laboral dels graduats a la Universitat Autònoma de Barcelona. Bellaterra, Cerdanyola del Vallès: ICE de la UAB.

MasjuAn, J.M.; TROIANO, H.; Molins, C. (2003). Els estudiants universitaris davant dels seus estudis i el futur professional. Aqu Catalunya (edición policopiada).

MCINNIS, CRAIG (2002). «Signs of Disengagement? Responding to the Changing Work and Study Patterns of Full Time Undergraduates in Australian Universities». En Enders, J.; Fulton, O. (eds.). Higher Education in a Globalising World. Dordrecht: Fluwer Academic Publishers, p. 175-191.

RodríGUez EspinaR, S. (dir.) (2003). Educació superior i treball a Catalunya. Barcelona: AQU Catalunya. 